\title{
Rogue Wave and Multiple Lump Solutions of the (2+1)-Dimensional Benjamin-Ono Equation in Fluid Mechanics
}

\author{
Zhonglong Zhao $\mathbb{D}$, Lingchao He, and Yubin Gao \\ Department of Mathematics, North University of China, Taiyuan, Shanxi 030051, China \\ Correspondence should be addressed to Zhonglong Zhao; zhaozlhit@163.com
}

Received 5 April 2019; Accepted 19 June 2019; Published 7 August 2019

Academic Editor: Dimitri Volchenkov

Copyright (c) 2019 Zhonglong Zhao et al. This is an open access article distributed under the Creative Commons Attribution License, which permits unrestricted use, distribution, and reproduction in any medium, provided the original work is properly cited.

\begin{abstract}
In this paper, the bilinear method is employed to investigate the rogue wave solutions and the rogue type multiple lump wave solutions of the $(2+1)$-dimensional Benjamin-Ono equation. Two theorems for constructing rogue wave solutions are proposed with the aid of a variable transformation. Four kinds of rogue wave solutions are obtained by means of Theorem 1 . In Theorem 2 , three polynomial functions are used to derive multiple lump wave solutions. The 3-lump solutions, 6-lump solutions, and 8lump solutions are presented, respectively. The 3-lump wave has a "triangular" structure. The centers of the 6-lump wave form a pentagram around a single lump wave. The 8-lump wave consists of a set of seven first order rogue waves and one second order rogue wave as the center. The multiple lump wave develops into low order rogue wave as parameters decline to zero. The method presented in this paper provides a uniform method for investigating high order rational solutions. All the results are useful in explaining high dimensional dynamical phenomena of the (2+1)-dimensional Benjamin-Ono equation.
\end{abstract}

\section{Introduction}

Rogue wave is an isolated huge wave, which plays an important role in analyzing many science problems, such as ocean's waves [1-3], optical fibers [4], Bose-Einstein condensates [5, $6]$, and financial markets $[7,8]$. The Darboux transformation method $[9,10]$ is one of the most effective methods to construct the rogue wave solutions of the integrable systems. Rogue wave solutions are an interesting class of lump-type solutions [11]. It is interesting that lump functions can provide approximate prototypes to model rogue waves [12]. Based on the bilinear method, the bilinear forms can be used to construct the lump solutions by choosing appropriate multivariate positive substitution quadratic functions. In 2015, $\mathrm{Ma}$ investigated the lump solutions of the $(2+1)$-dimensional Kadomtsev-Petviashvili equation by means of the bilinear forms and positive quadratic functions [13]. Inspired by this work, many researchers studied the lump solutions of the integrable systems, such as the $(2+1)$-dimensional Sawada-Kotera equation [14], the $(2+1)$-dimensional asymmetrical Nizhnik-Novikov-Veselov equation [15], the $(3+1)$ dimensional B-type KP equation [16], and the generalized
Calogero-Bogoyavlenskii-Schiff equation [17]. Furthermore, the generalized bilinear forms can also be used to derive the lump solutions [18].

Searching for the interaction solutions between the lump wave and other types of wave is one of the hot issues. There are many methods to investigate the lump solutions and the interaction solutions among solitons, such as the inverse scattering transformation [19], the Painlevé analysis [20-22], the symmetry analysis [23-27], and the Darboux transformation $[28,29]$. Fokas et al. studied the completely elastic collision between a lump wave and a line soliton of the DSII equation [30]. Tang et al. investigated the nonelastic collision between a lump wave and a stripe soliton of the $(2+1)$-dimensional Ito equation [31]. Then the interaction lump-soliton solutions $[32,33]$, lump-twin-stripe solutions [34], and lump-periodic wave solutions [35] were investigated.

Compared with the construction of single lump wave solutions, multiple wave solutions are more difficult to obtain. Zhang et al. obtained the multiple lump solutions of a $(3+1)$-dimensional nonlinear evolution equation by taking a "long wave" limit for the corresponding N-solitons [36]. 
The dynamical behaviors of the interactions of multilumps within the Kadomtsev-Petviashvili-1 equation were analyzed by numerical simulation [37]. The multiple lump solutions of a (3+1)-dimensional Boiti-Leon-Manna-Pempinelli equation in incompressible fluid were derived [38]. The high-order lumps of the $(3+1)$-dimensional KP-Boussinesq equation were presented by using Hirota's bilinear method [39]. Zhaqilao et al. proposed a new method to construct the multiple lump solutions with a controllable center [40, 41]. We constructed the multiple lump solutions of the $(3+1)$-dimensional potential Yu-Toda-Sasa-Fukuyama equation [42].

In this paper, we consider the $(2+1)$-dimensional Benjamin-Ono equation

$$
u_{t t}+a\left(u^{2}\right)_{x x}+b u_{x x x x}+u_{y y}=0
$$

where $u=u(x, y, t)$. Eq.(1) is the reduction of a generalized $(2+1)$-dimensional Boussinesq equation $[43,44]$. The $(2+1)$ dimensional Benjamin-Ono equation arises in the study of internal waves in deep stratified fluids [45].

The organization of the paper is as follows. In Section 2, multiple rogue wave solutions of (1) are constructed with the aid of bilinear method. A theorem is proposed to establish a unified approach to study multiple rogue wave solutions. In Section 3, three polynomial functions are introduced to construct the rogue type multiple lump wave solutions. The 3-lump, 6-lump, and 8-lump solutions are investigated in Sections 4, 5, and 6. Finally some conclusions are given in the last section.

\section{Multiple Rogue Wave Solutions}

In this section, we shall investigate rogue wave solutions of (1). Eq.(1) can be reduced to the following equation:

$$
m^{2} u_{X X}+a\left(u^{2}\right)_{X X}+b u_{X X X X}+u_{y y}=0,
$$

by setting $X=x+m t$. With the aid of variable transformation

$$
u=\frac{6 b}{a}(\ln f)_{X X}
$$

(2) can be reduced to the bilinear equation

$$
\begin{aligned}
& m^{2}\left(f f_{X X}-f_{X}{ }^{2}\right)+b\left(f f_{X X X X}-4 f_{X} f_{X X X}+3 f_{X X}{ }^{2}\right) \\
& +\left(f f_{y y}-f_{y}{ }^{2}\right)=0 .
\end{aligned}
$$

This bilinear form can be used to construct multiple rogue wave solutions of (1).

Theorem 1. (2+1)-Dimensional Benjamin-Ono equation (1) has the multiple rogue wave solutions

$$
u_{n}(x, y, t)=\frac{6 b}{a}(\ln f)_{X X}
$$

where

$$
\begin{aligned}
f(X, y) & =F_{n}(X, y) \\
& =\sum_{k=0}^{n(n+1) / 2} \sum_{i=0}^{k} a_{n(n+1)-2 k, 2 i} y^{2 i} X^{n(n+1)-2 k},
\end{aligned}
$$

$X=x+m t, f(X, y)$ is the solution of bilinear equation (4) and the coefficients $a_{n(n+1)-2 k, 2 i}$ are the parameters to be determined.

Theorem 1 provides a uniform method for constructing the multiple rogue wave solutions of (1). Using this method we obtain the following polynomials:

$$
\begin{aligned}
& F_{1}(X, y)=X^{2}+m^{2} y^{2}-3 \frac{b}{m^{2}}, \\
& F_{2}(X, y)=X^{6}-25 \frac{b X^{4}}{m^{2}}+3 m^{2} y^{2} X^{4}+\left(-125 \frac{b^{2}}{m^{4}}\right. \\
& \left.-90 b y^{2}+3 m^{4} y^{4}\right) X^{2}-1875 \frac{b^{3}}{m^{6}}+475 \frac{b^{2} y^{2}}{m^{2}} \\
& -17 b m^{2} y^{4}+m^{6} y^{6} \\
& F_{3}(X, y)=X^{12}+\left(-98 \frac{b}{m^{2}}+6 m^{2} y^{2}\right) X^{10}+\left(735 \frac{b^{2}}{m^{4}}\right. \\
& \left.-690 b y^{2}+15 m^{4} y^{4}\right) X^{8}+\left(-\frac{75460}{3} \frac{b^{3}}{m^{6}}\right. \\
& \left.+18620 \frac{b^{2} y^{2}}{m^{2}}-1540 b m^{2} y^{4}+20 m^{6} y^{6}\right) X^{6} \\
& +\left(-\frac{5187875}{3} \frac{b^{4}}{m^{8}}-220500 \frac{b^{3} y^{2}}{m^{4}}+37450 b^{2} y^{4}\right. \\
& \left.-1460 b m^{4} y^{6}+15 m^{8} y^{8}\right) X^{4}+\left(-\frac{159786550}{3} \frac{b^{5}}{m^{10}}\right. \\
& +565950 \frac{b^{4} y^{2}}{m^{6}}+14700 \frac{b^{3} y^{4}}{m^{2}}+35420 m^{2} b^{2} y^{6} \\
& \left.-570 b m^{6} y^{8}+6 m^{10} y^{10}\right) X^{2}+\frac{878826025}{9} \frac{b^{6}}{m^{12}} \\
& -\frac{300896750}{3} \frac{b^{5} y^{2}}{m^{8}}+\frac{16391725}{3} \frac{b^{4} y^{4}}{m^{4}}-\frac{798980}{3} \\
& \cdot b^{3} y^{6}+4335 b^{2} m^{4} y^{8}-58 b m^{8} y^{10}+m^{12} y^{12} \text {, } \\
& F_{4}(X, y)=X^{20}-270 \frac{b X^{18}}{m^{2}}+10 m^{2} y^{2} X^{18} \\
& +\left(16605 \frac{b^{2}}{m^{4}}-3030 b y^{2}+45 m^{4} y^{4}\right) X^{16} \\
& +\left(-351000 \frac{b^{3}}{m^{6}}+275400 \frac{b^{2} y^{2}}{m^{2}}-13800 b m^{2} y^{4}\right.
\end{aligned}
$$




$$
\begin{aligned}
& \left.+120 m^{6} y^{6}\right) X^{14}+\left(-18877950 \frac{b^{4}}{m^{8}}\right. \\
& -10621800 \frac{b^{3} y^{2}}{m^{4}}+1367100 b^{2} y^{4}-34440 b m^{4} y^{6} \\
& \left.+210 m^{8} y^{8}\right) X^{12}+\left(-2094264900 \frac{b^{5}}{m^{10}}\right. \\
& +107534700 \frac{b^{4} y^{2}}{m^{6}}-56586600 \frac{b^{3} y^{4}}{m^{2}} \\
& \left.+3308760 m^{2} b^{2} y^{6}-52500 b m^{6} y^{8}+252 m^{10} y^{10}\right) \\
& \cdot X^{10}+\left(-178095030750 \frac{b^{6}}{m^{12}}-4871002500 \frac{b^{5} y^{2}}{m^{8}}\right. \\
& +1071960750 \frac{b^{4} y^{4}}{m^{4}}-135286200 b^{3} y^{6} \\
& \left.+4513950 b^{2} m^{4} y^{8}-50820 b m^{8} y^{10}+210 m^{12} y^{12}\right) \\
& \cdot X^{8}+\left(-6967194507000 \frac{b^{7}}{m^{14}}\right. \\
& -521628471000 \frac{b^{6} y^{2}}{m^{10}}-636363000 \frac{b^{5} y^{4}}{m^{6}} \\
& +3824793000 \frac{b^{4} y^{6}}{m^{2}}-151237800 m^{2} b^{3} y^{8} \\
& +3601080 b^{2} m^{6} y^{10}-31080 b m^{10} y^{12} \\
& \left.+120 m^{14} y^{14}\right) X^{6}+\left(190578711448125 \frac{b^{8}}{m^{16}}\right. \\
& -33286514625000 \frac{b^{7} y^{2}}{m^{12}}-405853402500 \frac{b^{6} y^{4}}{m^{8}} \\
& -45237339000 \frac{b^{5} y^{6}}{m^{4}}+2667498750 b^{4} y^{8} \\
& -94613400 m^{4} b^{3} y^{10}+1619100 b^{2} m^{8} y^{12} \\
& \left.-11400 b m^{12} y^{14}+45 m^{16} y^{16}\right) X^{4} \\
& +\left(696163557521250 \frac{b^{9}}{m^{18}}\right. \\
& +870343420196250 \frac{b^{8} y^{2}}{m^{14}}-90898176915000 \frac{b^{7} y^{4}}{m^{10}} \\
& +1982064357000 \frac{b^{6} y^{6}}{m^{6}}-31477666500 \frac{b^{5} y^{8}}{m^{2}} \\
& +671510700 m^{2} b^{4} y^{10}-35645400 m^{6} b^{3} y^{12}
\end{aligned}
$$

$$
\begin{aligned}
& \left.+354600 b^{2} m^{10} y^{14}-2190 b m^{14} y^{16}+10 m^{18} y^{18}\right) \\
& \cdot X^{2}+5917390238930625 \frac{b^{10}}{m^{20}} \\
& -3474517664913750 \frac{b^{9} y^{2}}{m^{16}}+348683786758125 \\
& \cdot \frac{b^{8} y^{4}}{m^{12}}-43199536653000 \frac{b^{7} y^{6}}{m^{8}}+1200881855250 \\
& \cdot \frac{b^{6} y^{8}}{m^{4}}-21813668100 b^{5} y^{10}+360709650 b^{4} m^{4} y^{12} \\
& -3299400 m^{8} b^{3} y^{14}+23085 b^{2} m^{12} y^{16} \\
& -150 m^{16} b y^{18}+m^{20} y^{20},
\end{aligned}
$$

which corresponded to the rogue wave solutions $u_{1}, u_{2}, u_{3}$, and $u_{4}$.

Figures 1,2,3, and 4 show the dynamical behaviors of the rogue wave solutions $u_{1}, u_{2}, u_{3}$, and $u_{4}$, respectively.

Figures 1(a) and 1(b) show that the rogue wave $u_{1}$ has one peak which is higher than the water level. This kind of rogue wave is called lump wave that maintains the localization property in the planes $(x, y)$. The lump wave reaches the higher peak at the point $(-m t, 0)$. The lump wave Figures 1(a) and 1 (b) have two lower peaks. The coordinates of the lower peaks are $(-m t+3 \sqrt{-b} / m, 0)$ and $(-m t-3 \sqrt{-b} / m, 0)$. The center of the lump wave in the plane $(x, y)$ is $(-m t, 0)$. The one order rogue wave $u_{1}$ develops into one-soliton solution in Figure 1(c).

If we take $m=2, \alpha=\beta=-2, n=4$ in rogue wave $u_{2}$, we obtain the second order rogue wave described in Figures 2 (a) and 2(b). The rogue wave $u_{2}$ has the structure of twoline solitons in the plane $(x, t)$. Figures 3 and 4 plot the rogue waves $u_{3}$ and $u_{4}$. These show that the number of the higher peaks of the multiple rogue wave solutions is equivalent to the subscript of $u_{n}$. From Figures 1(a), 2(a), 3(a), and 4(a), we can conclude that the maxima of the solutions $u_{n}$ all lie on the line $y=0$. Figures 3(c) and 4(c) exhibit the fact that the rogue wave $u_{n}$ develops into the multiple soliton solutions in the plane $(x, t)$.

\section{Rogue Type Multiple Lump Wave Solutions}

Theorem 2. (2+1)-Dimensional Benjamin-Ono equation (1) has the rogue type multiple lump wave solutions

$$
u(x, y, t)=\frac{6 b}{a}(\ln f)_{X X},
$$

where

$$
\begin{aligned}
f(X, y)= & F_{n+1}(X, y)+2 \alpha y P_{n}(X, y) \\
& +2 \beta X Q_{n}(X, y)
\end{aligned}
$$




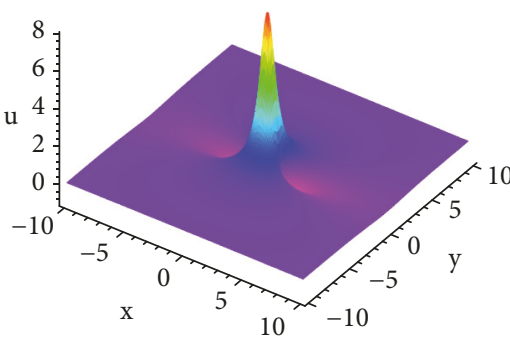

(a)

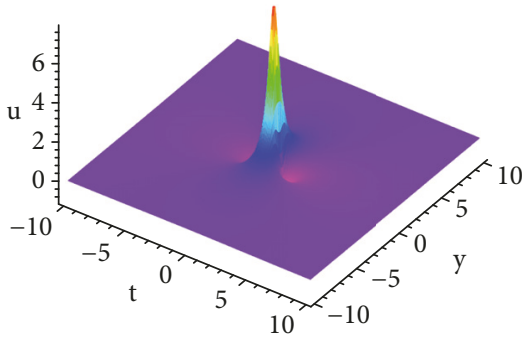

(b)

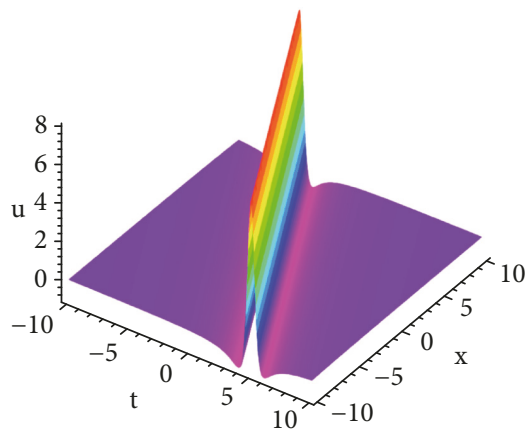

(c)

FIGURE 1: Plots (colored online) of rogue wave $u_{1}$ with $m=2, a=b=-2$. (a) Perspective view of the wave $u(x, y, 0)$, (b) perspective view of the wave $u(0, y, t)$, and (c) perspective view of the wave $u(x, 0, t)$.

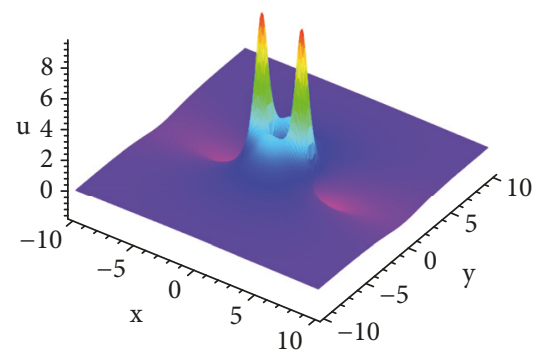

(a)

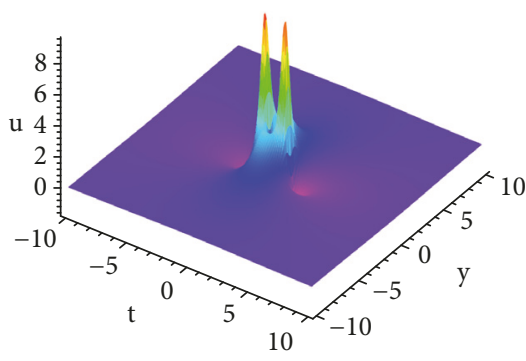

(b)

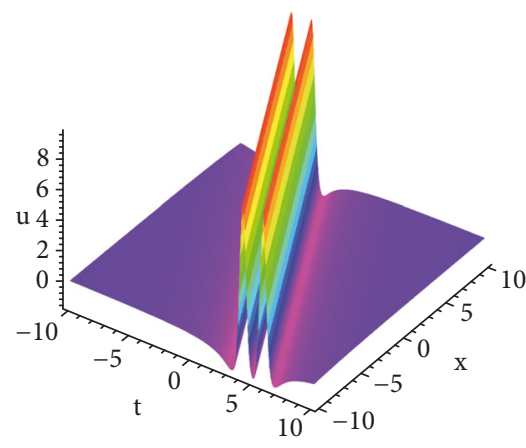

(c)

Figure 2: Plots (colored online) of rogue wave $u_{2}$ with $m=2, a=b=-2$. (a) Perspective view of the wave $u(x, y, 0)$, (b) perspective view of the wave $u(0, y, t)$, and (c) perspective view of the wave $u(x, 0, t)$.

$$
\begin{array}{r}
+\left(\alpha^{2}+\beta^{2}\right) F_{n-1}(X, y), \quad n \geq 2, \\
F_{n}(X, y)=\sum_{k=0}^{n(n+1) / 2} \sum_{i=0}^{k} a_{n(n+1)-2 k, 2 i} y^{2 i} X^{n(n+1)-2 k}, \\
P_{n}(X, y)=\sum_{k=0}^{n(n+1) / 2} \sum_{i=0}^{k} b_{n(n+1)-2 k, 2 i} X^{2 i} y^{n(n+1)-2 k}, \\
Q_{n}(X, y)=\sum_{k=0}^{n(n+1) / 2} \sum_{i=0}^{k} c_{n(n+1)-2 k, 2 i} y^{2 i} X^{n(n+1)-2 k},
\end{array}
$$

$X=x+m t, f(X, y)$ is the solution of bilinear equation (4), the coefficients $a_{n(n+1)-2 k, 2 i}, b_{n(n+1)-2 k, 2 i}$ and $c_{n(n+1)-2 k, 2 i}$ are the parameters to be determined, and $\alpha$ and $\beta$ are arbitrary constants.

Remark 3. Theorem 2 gives a uniform method for constructing the rogue type multiple lump wave solutions of (1). One can obtain the multiple wave solutions more than six lump waves by selecting $n \geq 3$. The parameters $\alpha$ and $\beta$ determine the shape of the multiple lump wave. The multiple lump wave tends to a low order rogue wave as $\alpha^{2}+\beta^{2}$ declines to 0 . All peaks of the lump wave tend to the same height when $\alpha^{2}+\beta^{2}$ is sufficiently large. In what follows, we shall construct the multiple lump solutions of (1).

\section{3-Lump Solutions}

Based on Theorem 2, we construct the 3-lump solutions with the aid of following polynomial functions:

$$
\begin{aligned}
f(X, y)= & F_{2}(X, y)+2 \alpha y P_{1}(X, y)+2 \beta X Q_{1}(X, y) \\
& +\left(\alpha^{2}+\beta^{2}\right) \\
= & X^{6}+a_{4,0} X^{4}+a_{4,2} X^{4} y^{2} \\
& +\left(a_{2,0}+a_{2,2} y^{2}+a_{2,4} y^{4}\right) X^{2}+a_{0,0} \\
& +a_{0,2} y^{2}+a_{0,4} y^{4}+a_{0,6} y^{6} \\
& +2 \alpha y\left(b_{0,0}+b_{2,0} y^{2}+b_{0,2} X^{2}\right) \\
& +2 \beta X\left(c_{0,0}+c_{0,2} y^{2}+c_{2,0} X^{2}\right)+\alpha^{2} \\
& +\beta^{2}
\end{aligned}
$$




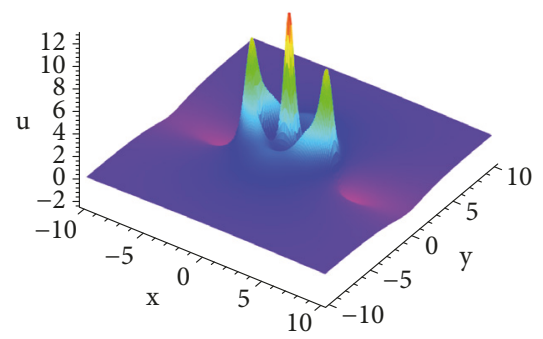

(a)

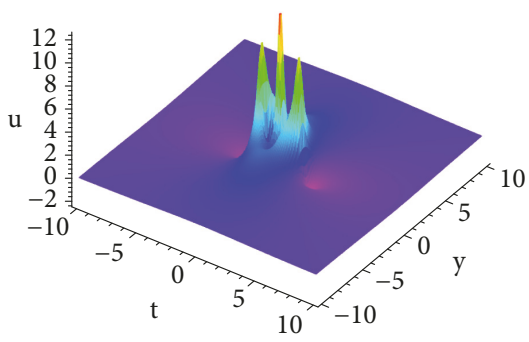

(b)

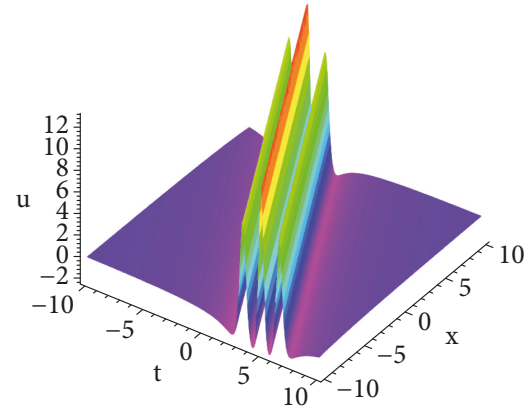

(c)

Figure 3: Plots (colored online) of rogue wave $u_{3}$ with $m=2, a=b=-2$. (a) Perspective view of the wave $u(x, y, 0)$, (b) perspective view of the wave $u(0, y, t)$, and (c) perspective view of the wave $u(x, 0, t)$.

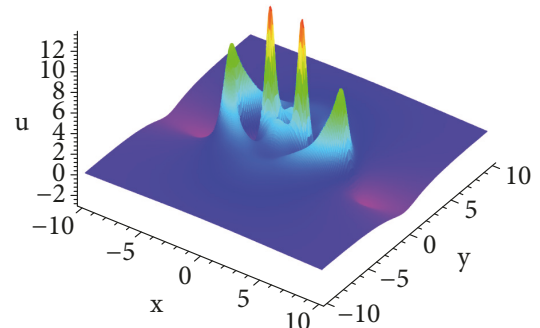

(a)

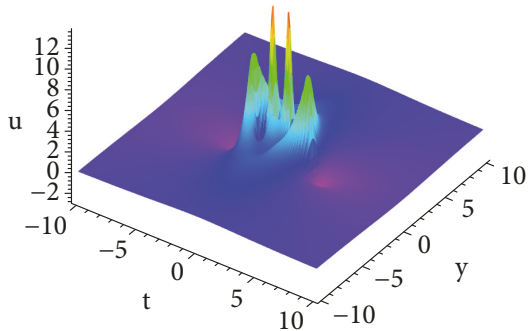

(b)

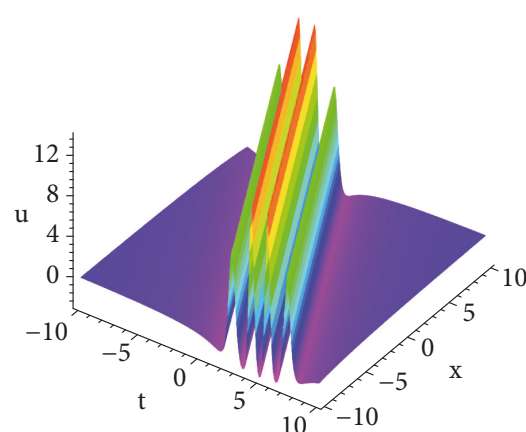

(c)

Figure 4: Plots (colored online) of rogue wave $u_{4}$ with $m=2, a=b=-2$. (a) Perspective view of the wave $u(x, y, 0)$, (b) perspective view of the wave $u(0, y, t)$, and (c) perspective view of the wave $u(x, 0, t)$.

Substituting (11) into (4) and setting all the coefficients of the different polynomials of $X$ and $y$ to zero yield a set of determining equations. Solving these equations, one has

$$
\begin{aligned}
& a_{0,0} \\
& =-\frac{16875 b^{3}+9 m^{6} \alpha^{2}+9 m^{6} \beta^{2}-\alpha^{2} m^{4} b_{0,2}^{2}-9 m^{6} \beta^{2} c_{2,0}^{2}}{9 m^{6}} \\
& a_{0,2}=\frac{475 b^{2}}{m^{2}}, \\
& a_{0,4}=-17 b m^{2} \\
& a_{0,6}=m^{6} \\
& a_{2,0}=-\frac{125 b^{2}}{m^{4}} \\
& a_{2,2}=-90 b \\
& a_{2,4}=3 m^{4} \\
& a_{4,0}=-\frac{25 b}{m^{2}} \\
& a_{4,2}=3 m^{2}
\end{aligned}
$$

$$
\begin{aligned}
& b_{0,0}=-\frac{5 b_{0,2} b}{3 m^{2}}, \\
& b_{2,0}=-\frac{m^{2} b_{0,2}}{3}, \\
& c_{0,0}=\frac{c_{2,0} b}{m^{2}}, \\
& c_{0,2}=-3 c_{2,0} m^{2} .
\end{aligned}
$$

Then we obtain the 3-lump solutions

$$
u(x, y, t)=\frac{6 b}{a}(\ln f)_{X X}
$$

where $X=x+m t$, the coefficients of $f$ are determined by (12), and $m, a, b, \alpha$, and $\beta$ are arbitrary real constants. Figure 5 plots the 3-lump wave (13) when $m=2, a=b=-2, b_{0,2}=c_{2,0}=1$, $\alpha=3000, \beta=4000$. The 3 -lump wave is the arrangement of three first order rogue waves in the planes $(x, y)$ and $(y, t)$, which forms a structure of triangular. From Figures 5(c) and 5(f), we observe that 3-lump wave solution (13) develops into two-soliton solution in the plane $(x, t)$. 


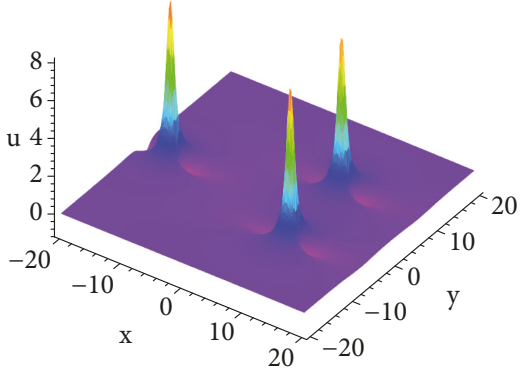

(a)

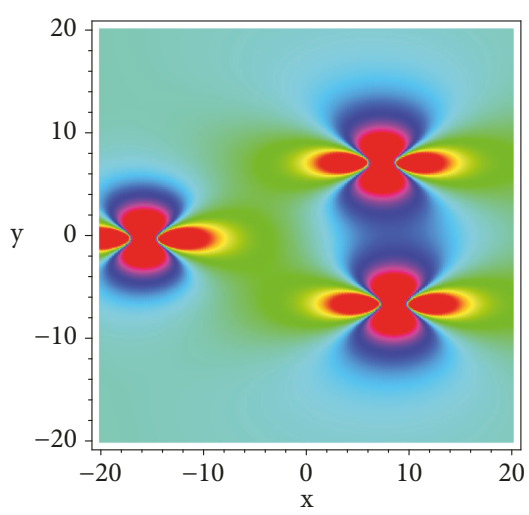

(d)

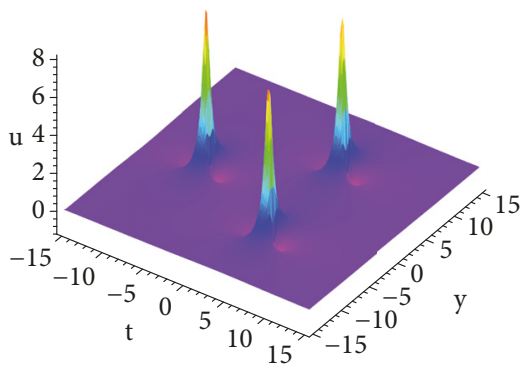

(b)

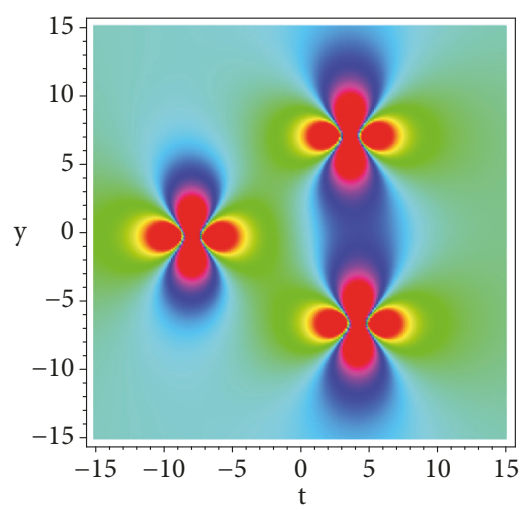

(e)

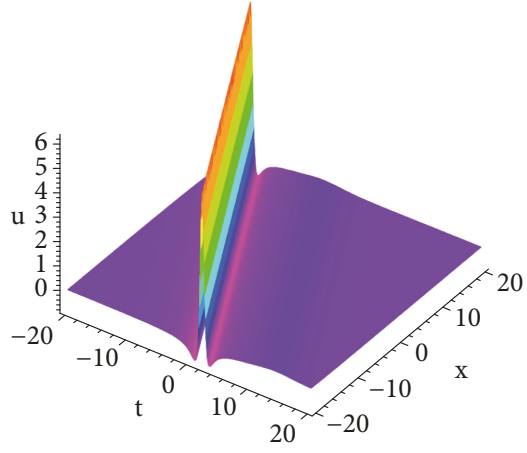

(c)

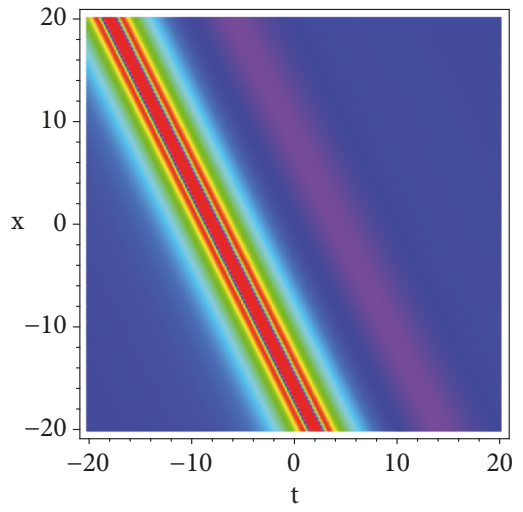

(f)

Figure 5: Plots (colored online) of 3-lump wave $u$ (13) with $m=2, a=b=-2, b_{0,2}=c_{2,0}=1, \alpha=3000, \beta=4000$. (a) Perspective view of the wave $u(x, y, 0)$, (b) perspective view of the wave $u(0, y, t)$, (c) perspective view of the wave $u(x, 0, t)$, (d) overhead view of the wave (a), (e) overhead view of the wave (b), and (f) overhead view of the wave (c).

\section{6-Lump Solutions}

In order to obtain the 6-lump solutions of (1), we choose

$$
\begin{aligned}
f(X, y)= & F_{3}(X, y)+2 \alpha y P_{2}(X, y)+2 \beta X Q_{2}(X, y) \\
& +\left(\alpha^{2}+\beta^{2}\right) F_{1}(X, y)
\end{aligned}
$$

where

$$
\begin{gathered}
F_{3}(X, y)=X^{12}+\left(a_{10,0}+a_{10,2} y^{2}\right) X^{10}+\left(a_{8,0}\right. \\
\left.+a_{8,2} y^{2}+a_{8,4} y^{4}\right) X^{8}+\left(a_{6,0}+a_{6,2} y^{2}+a_{6,4} y^{4}\right. \\
\left.+a_{6,6} y^{6}\right) X^{6}+\left(a_{4,0}+a_{4,2} y^{2}+a_{4,4} y^{4}+a_{4,6} y^{6}\right. \\
\left.+a_{4,8} y^{8}\right) X^{4}+\left(a_{2,0}+a_{2,2} z^{2}+a_{2,4} y^{4}+a_{2,6} y^{6}\right. \\
\left.+a_{2,8} y^{8}+a_{2,10} y^{10}\right) X^{2}+a_{0,0}+a_{0,2} y^{2}+a_{0,4} y^{4} \\
+a_{0,6} y^{6}+a_{0,8} y^{8}+a_{0,10} y^{10}+a_{0,12} y^{12}, \\
P_{2}(X, y)=b_{0,0}+\left(b_{2,0}+b_{2,2} X^{2}+b_{2,4} X^{4}\right) y^{2}+\left(b_{4,0}\right. \\
\left.+b_{4,2} X^{2}\right) y^{4}+y^{6}+b_{0,2} X^{2}+b_{0,4} X^{4}+b_{0,6} X^{6},
\end{gathered}
$$

$$
\begin{aligned}
& Q_{2}(X, y)=c_{0,0}+c_{0,2} y^{2}+c_{0,4} y^{4}+c_{0,6} y^{6}+\left(c_{2,0}\right. \\
& \left.\quad+c_{2,2} y^{2}+c_{2,4} y^{4}\right) X^{2}+\left(c_{4,0}+c_{4,2} y^{2}\right) X^{4}+X^{6} \\
& F_{1}(X, y)=X^{2}+a_{0,2} y^{2}+a_{0,0} .
\end{aligned}
$$

Substituting (14) into (4) and setting all the coefficients of the different polynomials of $X$ and $y$ to zero yield a set of determining equations. Solving these equations, one has

$$
\begin{aligned}
& a_{0,0}=\frac{b\left(878826025 m^{4} b^{2}-27 \alpha^{2}-27 m^{14} \beta^{2}\right)}{9 m^{16}\left(\alpha^{2}+\beta^{2}+1\right)}, \\
& a_{0,2}=-\frac{300896750 m^{4} b^{5}-3 \alpha^{2}-3 m^{14} \beta^{2}}{3 m^{12}\left(\alpha^{2}+\beta^{2}+1\right)}, \\
& a_{0,4}=\frac{16391725 b^{4}}{3 m^{4}}, \\
& a_{0,6}=-\frac{798980 b^{3}}{3}, \\
& a_{0,8}=4335 b^{2} m^{4},
\end{aligned}
$$




$$
\begin{aligned}
& a_{0,10}=-58 \mathrm{bm}^{8}, \\
& a_{0,12}=m^{12}, \\
& a_{2,0}=-\frac{-3 \alpha^{2}+3 m^{14} \alpha^{2}+159786550 m^{4} b^{5}}{3 m^{14}}, \\
& a_{2,2}=\frac{565960 b^{4}}{m^{6}}, \\
& a_{2,4}=\frac{14700 b^{3}}{m^{2}}, \\
& a_{2,6}=35420 b^{2} m^{2} \text {, } \\
& a_{2,8}=-570 b m^{6} \text {, } \\
& a_{2,10}=6 m^{10} \text {, } \\
& a_{4,0}=-\frac{5187875 b^{4}}{3 m^{8}}, \\
& a_{4,2}=-\frac{220500 b^{3}}{m^{4}}, \\
& a_{4,4}=37450 b^{2} \text {, } \\
& a_{4,6}=-1460 \mathrm{bm}^{4}, \\
& a_{4,8}=15 m^{8}, \\
& a_{6,0}=-\frac{75460 b^{3}}{3 m^{6}}, \\
& a_{6,2}=\frac{18620 b^{2}}{m^{2}}, \\
& a_{6,4}=-1540 \mathrm{bm}^{2}, \\
& a_{6,6}=20 m^{6} \text {, } \\
& a_{8,0}=\frac{735 b^{2}}{m^{4}}, \\
& a_{8,2}=-690 b \text {, } \\
& a_{8,4}=15 m^{4} \text {, } \\
& a_{10,0}=-\frac{-98 b}{m^{2}} \text {, } \\
& a_{10,2}=6 m^{2} \text {, } \\
& b_{0,0}=-\frac{18865 b^{3}}{3 m^{12}}, \\
& b_{0,2}=-\frac{665 b^{2}}{m^{10}} \text {, } \\
& b_{0,4}=-\frac{105 b}{m^{8}} \text {, } \\
& b_{0,6}=\frac{5}{m^{6}} \text {, }
\end{aligned}
$$

$$
\begin{aligned}
& b_{2,0}=-\frac{245 b^{2}}{m^{8}}, \\
& b_{2,2}=\frac{190 b}{m^{6}}, \\
& b_{2,4}=-\frac{5}{m^{4}}, \\
& b_{4,0}=\frac{7 b}{m^{4}}, \\
& b_{4,2}=-\frac{9}{m^{2}}, \\
& c_{0,0}=-\frac{12005 b^{3}}{3 m^{6}}, \\
& c_{0,2}=\frac{535 b^{2}}{m^{2}}, \\
& c_{0,4}=-45 b m^{2}, \\
& c_{0,6}=5 m^{6}, \\
& c_{2,0}=-\frac{245 b^{2}}{m^{4}}, \\
& c_{2,2}=230 b, \\
& c_{2,4}=-5 m^{4}, \\
& c_{4,0}=-\frac{13 b}{m^{2}}, \\
& c_{4,2}=-9 m^{2} .
\end{aligned}
$$

Then we obtain the 6-lump solutions

$$
u(x, y, t)=\frac{6 b}{a}(\ln f)_{X X}
$$

where $X=x+m t$ and the coefficients of $f$ are determined by (16), $m, a, b, \alpha$, and $\beta$ are arbitrary real constants.

Figures 6(a) and 6(e) plot 6-lump wave (17) when $m=$ 2, $a=b=-2, \alpha=8, \beta=10$. This case illustrates that as $\alpha^{2}+\beta^{2}$ tends to 0 the six lump wave becomes a third order rogue wave $u_{3}$ in Section 2. Figures 6(b), 6(c), 6(f), and 6(g) exhibit the fact that three more peaks appear as $\alpha$ and $\beta$ increase. As $\alpha$ and $\beta$ become more large, six lump waves appear and all the centers of the single lump waves form a structure of pentagram. Figure 6(d) shows that all the peaks of the 6-lump wave tend to the same hight when $\alpha^{2}+\beta^{2}$ is sufficiently large. Figure 7 plots the 6-lump wave (17) when $m=2, a=b=-2$, $\alpha=8 \times 10^{5}, \beta=10 \times 10^{5}$. The corresponding 6-lump wave is composed of six single lump waves, which array a pentagon. Figure 7(c) shows that the 6-lump wave solutions have the structure of three solitons in the plane $(x, t)$. 


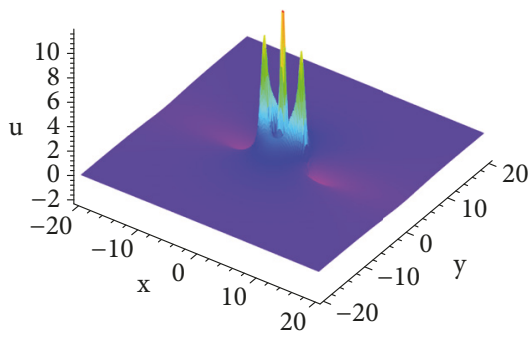

(a)

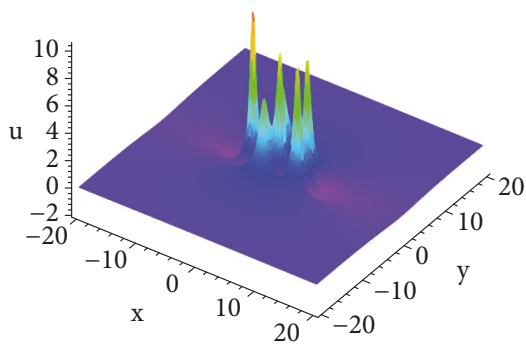

(c)

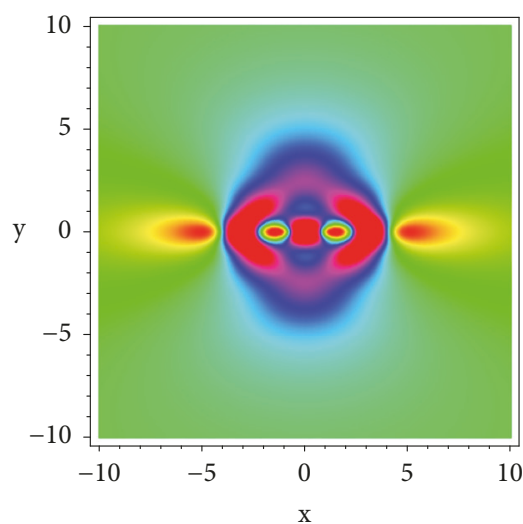

(e)

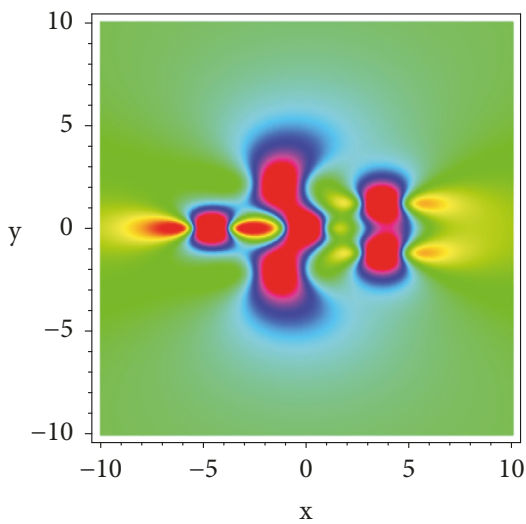

(g)

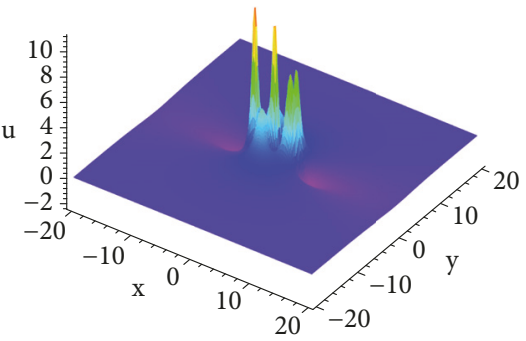

(b)

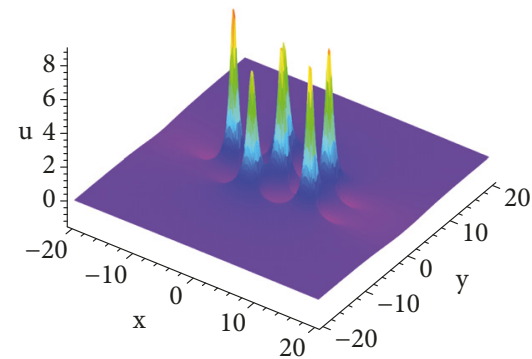

(d)

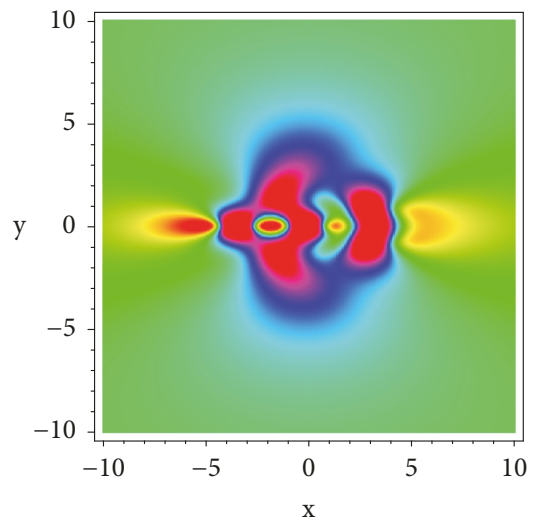

(f)

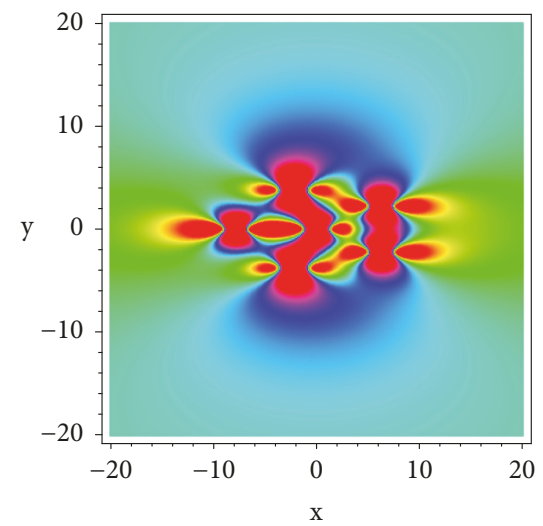

(h)

FIGURE 6: Plots (colored online) of 6-lump wave $u$ (17) with $m=2, a=b=-2$. (a) Perspective view of the wave $u(x, y, 0)$ with $\alpha=8, \beta=10$, (b) perspective view of the wave $u(x, y, 0)$ with $\alpha=800, \beta=1000$, (c) perspective view of the wave $u(x, y, 0)$ with $\alpha=3000$, $\beta=4000$, (d) perspective view of the wave $u(x, y, 0)$ with $\alpha=3 \times 10^{4}, \beta=4 \times 10^{4}$, (e) overhead view of the wave (a), (f) overhead view of the wave (b), (g) overhead view of the wave (c), and (h) overhead view of the wave $(\mathrm{d})$. 


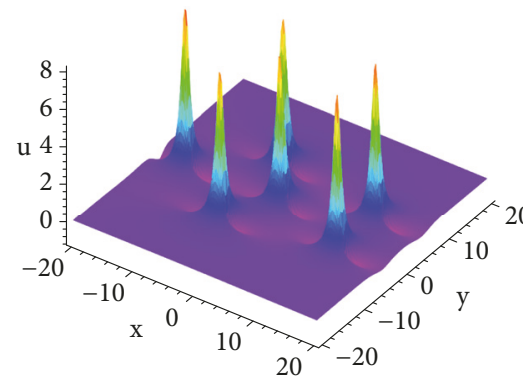

(a)

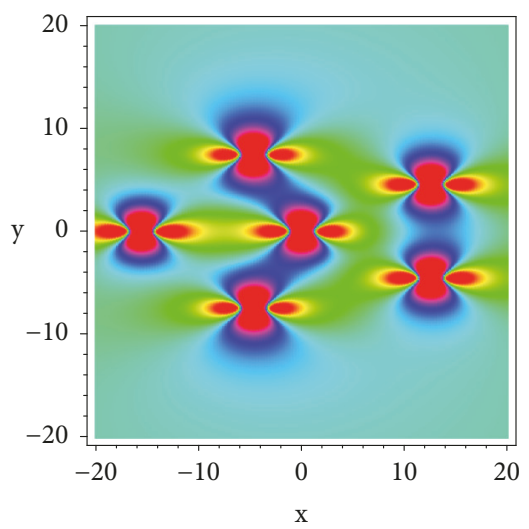

(d)

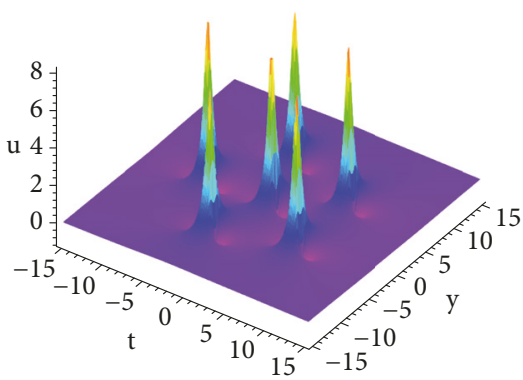

(b)

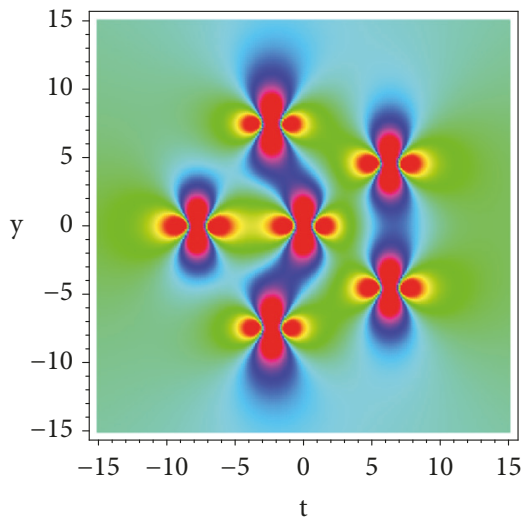

(e)

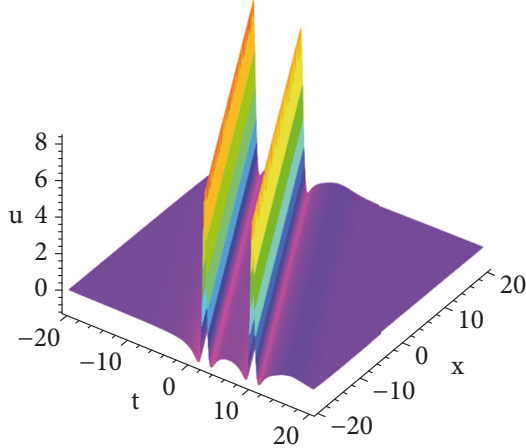

(c)

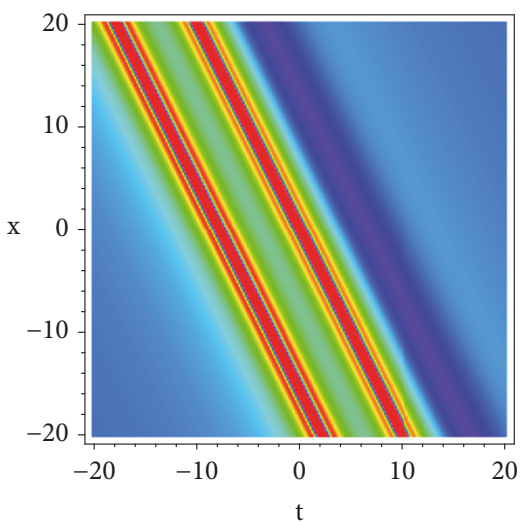

(f)

Figure 7: Plots (colored online) of 6-lump wave $u$ (17) with $m=2, a=b=-2, \alpha=8 \times 10^{5}, \beta=10 \times 10^{5}$. (a) Perspective view of the wave $u(x, y, 0),(\mathrm{b})$ perspective view of the wave $u(0, y, t),(\mathrm{c})$ perspective view of the wave $u(x, 0, t)$, (d) overhead view of the wave (a), (e) overhead view of the wave (b), and (f) overhead view of the wave (c).

\section{8-Lump Solutions}

To find the 8-lump solutions of (1), we investigate polynomial function solutions to (4) with the assumption

$$
\begin{aligned}
f(X, y)= & F_{4}(X, y)+2 \alpha y P_{3}(X, y)+2 \beta X Q_{3}(X, y) \\
& +\left(\alpha^{2}+\beta^{2}\right) F_{2}(X, y),
\end{aligned}
$$

where

$$
\begin{aligned}
& F_{4}(X, y)=X^{20}+\left(a_{18,0}+a_{18,2} y^{2}\right) X^{18}+\left(a_{16,0}\right. \\
& \left.+a_{16,2} y^{2}+a_{16,4} y^{4}\right) X^{16}+\left(a_{14,0}+a_{14,2} y^{2}+a_{14,4} y^{4}\right. \\
& \left.+a_{14,6} y^{6}\right) X^{14}+\left(a_{12,0}+a_{12,2} y^{2}+a_{12,4} y^{4}+a_{12,6} y^{6}\right. \\
& \left.+a_{12,8} y^{8}\right) X^{12}+\left(a_{10,0}+a_{10,2} y^{2}+a_{10,4} y^{4}+a_{10,6} y^{6}\right. \\
& \left.+a_{10,8} y^{8}+a_{10,10} y^{10}\right) X^{10}+\left(a_{8,0}+a_{8,2} y^{2}+a_{8,4} y^{4}\right. \\
& \left.+a_{8,6} y^{6}+a_{8,8} y^{8}+a_{8,10} y^{10}+a_{8,12} y^{12}\right) X^{8}+\left(a_{6,0}\right. \\
& +a_{6,2} y^{2}+a_{6,4} y^{4}+a_{6,6} y^{6}+a_{6,8} y^{8}+a_{6,10} y^{10}
\end{aligned}
$$

$$
\begin{aligned}
& \left.+a_{6,12} y^{12}+a_{6,14} y^{14}\right) X^{6}+\left(a_{4,0}+a_{4,2} y^{2}+a_{4,4} y^{4}\right. \\
& +a_{4,6} y^{6}+a_{4,8} y^{8}+a_{4,10} y^{10}+a_{4,12} y^{12}+a_{4,14} y^{14} \\
& \left.+a_{4,16} y^{16}\right) X^{4}+\left(a_{2,0}+a_{2,2} y^{2}+a_{2,4} y^{4}+a_{2,6} y^{6}\right. \\
& +a_{2,8} y^{8}+a_{2,10} y^{10}+a_{2,12} y^{12}+a_{2,14} y^{14}+a_{2,16} y^{16} \\
& \left.+a_{2,18} y^{18}\right) X^{2}+a_{0,0}+a_{0,2} y^{2}+a_{0,4} y^{4}+a_{0,6} y^{6} \\
& +a_{0,8} y^{8}+a_{0,10} y^{10}+a_{0,12} y^{12}+a_{0,14} y^{14}+a_{0,16} y^{16} \\
& +a_{0,18} y^{18}+a_{0,20} y^{20} \\
& P_{3}(X, y)=b_{12,0} y^{12}+\left(b_{10,0}+b_{10,2} X^{2}\right) y^{10}+\left(b_{8,0}\right. \\
& \left.+b_{8,2} X^{2}+b_{8,4} X^{4}\right) y^{8}+\left(b_{6,0}+b_{6,2} X^{2}+b_{6,4} X^{4}\right. \\
& \left.+b_{6,6} X^{6}\right) y^{6}+\left(b_{4,0}+b_{4,2} X^{2}+b_{4,4} X^{4}+b_{4,6} X^{6}\right. \\
& \left.+b_{4,8} X^{8}\right) y^{4}+\left(b_{2,0}+b_{2,2} X^{2}+b_{2,4} X^{4}+b_{2,6} X^{6}\right. \\
& \left.+b_{2,8} X^{8}+b_{2,10} X^{10}\right) y^{2}+b_{0,0}+b_{0,2} X^{2}+b_{0,4} X^{4} \\
& +b_{0,6} X^{6}+b_{0,8} X^{8}+b_{0,10} X^{10}+b_{0,12} X^{12}
\end{aligned}
$$




$$
\begin{aligned}
& Q_{3}(X, y)=c_{12,0} X^{12}+\left(c_{10,0}+c_{10,2} y^{2}\right) X^{10}+\left(c_{8,0}\right. \\
& +c_{0,6} y^{6}+c_{0,8} y^{8}+c_{0,10} y^{10}+c_{0,12} y^{12}, \\
& \left.+c_{8,2} y^{2}+c_{8,4} y^{4}\right) X^{8}+\left(c_{6,0}+c_{6,2} y^{2}+c_{6,4} y^{4}\right. \\
& \left.+c_{6,6} y^{6}\right) X^{6}+\left(c_{4,0}+c_{4,2} y^{2}+c_{4,4} y^{4}+c_{4,6} y^{6}\right. \\
& F_{2}(X, y)=X^{6}+\left(a_{4,0}+a_{4,2} y^{2}\right) X^{4}+\left(a_{2,0}+a_{2,2} y^{2}\right. \\
& \left.+a_{2,4} y^{4}\right) X^{2}+a_{0,0}+a_{0,2} y^{2}+a_{0,4} y^{4}+a_{0,6} y^{6}, \\
& \left.+c_{4,8} y^{8}\right) X^{4}+\left(c_{2,0}+c_{2,2} y^{2}+c_{2,4} y^{4}+c_{2,6} y^{6}\right. \\
& \left.+c_{2,8} y^{8}+c_{2,10} y^{10}\right) X^{2}+c_{0,0}+c_{0,2} y^{2}+c_{0,4} y^{4} \\
& a_{0,0}=\frac{a_{18,0}{ }^{3}\left(5727449317681 a_{18,0}{ }^{7} m^{2}+3874204890000000 \alpha^{2} b_{0,12}{ }^{2}+189836039610000000 m^{2} \beta^{2} c_{12,0}{ }^{2}\right)}{1992822809409936000000 m^{2}\left(1+\alpha^{2}+\beta^{2}\right)}, \\
& a_{0,2}=\frac{a_{18,0}{ }^{2}\left(490732619400000 \alpha^{2} b_{0,12}{ }^{2}+1681494968699 a_{18,0}{ }^{7} m^{2}+24045898350600000 m^{2} \beta^{2} c_{12,0}^{2}\right)}{3690412610018400000\left(1+\alpha^{2}+\beta^{2}\right)}, \\
& a_{0,4}=\frac{m^{2} a_{18,0}\left(337491467677 a_{18,0}{ }^{7} m^{2}+35126124336000 \alpha^{2} b_{0,12}{ }^{2}+1721180092464000 m^{2} \beta^{2} c_{12,0}{ }^{2}\right)}{27336389703840000\left(1+\alpha^{2}+\beta^{2}\right)} \text {, } \\
& a_{0,6}=\frac{m^{4}\left(21092893290000 m^{2} \beta^{2} c_{12,0}{ }^{2}+8711017679 a_{18,0}{ }^{7} m^{2}+430467210000 \alpha^{2} b_{0,12}{ }^{2}\right)}{21092893290000\left(1+\alpha^{2}+\beta^{2}\right)}, \\
& a_{0,8}=\frac{177908423}{57395628000} a_{18,0}{ }^{6} \mathrm{~m}^{8} \text {, } \\
& a_{0,10}=\frac{24237409}{1594323000} m^{10} a_{18,0}{ }^{5}, \\
& a_{0,12}=\frac{801577}{11809800} m^{12} a_{18,0}^{4}, \\
& a_{0,14}=\frac{611}{3645} m^{14} a_{18,0}{ }^{3} \\
& a_{0,16}=\frac{19}{60} m^{16} a_{18,0}^{2}, \\
& a_{0,18}=\frac{5}{9} m^{18} a_{18,0} \\
& a_{0,20}=m^{20} \text {, } \\
& a_{2,0}=\frac{a_{18,0}{ }^{2}\left(336908783393 a_{18,0}{ }^{7} m^{2}+129140163000000 \alpha^{2} b_{0,12}{ }^{2}+6327867987000000 m^{2} \beta^{2} c_{12,0}{ }^{2}\right)}{3690412610018400000 m^{2}\left(1+\alpha^{2}+\beta^{2}\right)}, \\
& a_{2,2}=\frac{a_{18,0}\left(421203235453 a_{18,0}{ }^{7} m^{2}+92980917360000 \alpha^{2} b_{0,12}{ }^{2}+4556064950640000 m^{2} \beta^{2} c_{12,0}^{2}\right)}{13668194851920000\left(1+\alpha^{2}+\beta^{2}\right)}, \\
& a_{2,4}=\frac{\left(37967207922000 m^{2} \beta^{2} c_{12,0}{ }^{2}+10997557207 a_{18,0}{ }^{7} m^{2}+774840978000 \alpha^{2} b_{0,12}{ }^{2}\right) m^{2}}{12655735974000\left(1+\alpha^{2}+\beta^{2}\right)}, \\
& a_{2,6}=\frac{73409791}{14348907000} m^{6} a_{18,0}{ }^{6}, \\
& a_{2,8}=\frac{2331679}{106288200} m^{8} a_{18,0}{ }^{5} \text {, } \\
& a_{2,10}=\frac{746123}{5904900} m^{10} a_{18,0}{ }^{4} \text {, } \\
& a_{2,12}=\frac{6601}{3645} m^{12} a_{18,0}{ }^{3},
\end{aligned}
$$

Submitting (18) into (4), we obtain a set of constraining 


$$
\begin{aligned}
& a_{2,14}=\frac{394}{81} m^{14} a_{18,0}^{2} \\
& a_{2,16}=\frac{73}{9} m^{16} a_{18,0} \\
& a_{2,18}=10 m^{18} \text {, } \\
& a_{4,0}=\frac{a_{18,0}\left(184461370093 a_{18,0}{ }^{7} m^{2}+51656065200000 \alpha^{2} b_{0,12}{ }^{2}+2531147194800000 m^{2} \beta^{2} c_{12,0}{ }^{2}\right)}{27336389703840000 m^{2}\left(1+\alpha^{2}+\beta^{2}\right)}, \\
& a_{4,2}=\frac{161090293 a_{18,0}{ }^{7} m^{2}+30993639120 \alpha^{2} b_{0,12}{ }^{2}+1518688316880 m^{2} \beta^{2} c_{12,0}{ }^{2}}{506229438960\left(1+\alpha^{2}+\beta^{2}\right)}, \\
& a_{4,4}=-\frac{6012643}{5739562800} a_{18,0}{ }^{6} \mathrm{~m}^{4}, \\
& a_{4,6}=\frac{1675457}{53144100} m^{6} a_{18,0}^{5}, \\
& a_{4,8}=\frac{79037}{157464} m^{8} a_{18,0}^{4}, \\
& a_{4,10}=\frac{17521}{3645} a_{18,0}^{3} m^{10} \\
& a_{4,12}=\frac{1799}{81} a_{18,0}^{2} m^{12} \\
& a_{4,14}=\frac{380}{9} m^{14} a_{18,0} \\
& a_{4,16}=45 m^{16} \text {, } \\
& a_{6,0}=-\left(63278679870000 m^{2} \alpha^{2}-1291401630000 \alpha^{2} b_{0,12}^{2}-63278679870000 m^{2} \beta^{2} c_{12,0}{ }^{2}+63278679870000 m^{2} \beta^{2}\right. \\
& \left.-4214722603 a_{18,0}{ }^{7} m^{2}\right) \frac{1}{63278679870000 m^{2}}, \\
& a_{6,2}=-\frac{19319573}{14348907000} a_{18,0}{ }^{6} \mathrm{~m}^{2} \text {, } \\
& a_{6,4}=\frac{23569}{53144100} m^{4} a_{18,0}{ }^{5}, \\
& a_{6,6}=\frac{141659}{196830} m^{6} a_{18,0}^{4}, \\
& a_{6,8}=\frac{28007}{3645} m^{8} a_{18,0}{ }^{3}, \\
& a_{6,10}=\frac{20006}{405} m^{10} a_{18,0}{ }^{2}, \\
& a_{6,12}=\frac{1036}{9} m^{12} a_{18,0}, \\
& a_{6,14}=120 m^{14} \text {, } \\
& a_{8,0}=-\frac{26384449}{57395628000} a_{18,0}{ }^{6}, \\
& a_{8,2}=\frac{72163}{21257640} a_{18,0}{ }^{5} \mathrm{~m}^{2}, \\
& a_{8,4}=\frac{158809}{787320} m^{4} a_{18,0}{ }^{4}, \\
& a_{8,6}=\frac{8351}{1215} a_{18,0}^{3} \mathrm{~m}^{6},
\end{aligned}
$$




$$
\begin{aligned}
& a_{8,8}=\frac{10031}{162} m^{8} a_{18,0}{ }^{2}, \\
& a_{8,10}=\frac{1694}{9} m^{10} a_{18,0}, \\
& a_{8,12}=210 m^{12} \text {, } \\
& a_{10,0}=\frac{2326961}{1594323000} a_{18,0}{ }^{5} \text {, } \\
& a_{10,2}=\frac{119483}{5904900} a_{18,0}{ }^{4} m^{2} \text {, } \\
& a_{10,4}=\frac{3493}{1215} m^{4} a_{18,0}{ }^{3}, \\
& a_{10,6}=\frac{18382}{405} m^{6} a_{18,0}{ }^{2} \text {, } \\
& a_{10,8}=\frac{1750}{9} m^{8} a_{18,0}, \\
& a_{10,10}=252 m^{10} \text {, } \\
& a_{12,0}=-\frac{41951}{11809800} a_{18,0}{ }^{4} \text {, } \\
& a_{12,2}=\frac{1967}{3645} a_{18,0}{ }^{3} \mathrm{~m}^{2}, \\
& a_{12,4}=\frac{1519}{81} m^{4} a_{18,0}{ }^{2} \text {, } \\
& a_{12,6}=\frac{1148}{9} m^{6} a_{18,0}, \\
& a_{12,8}=210 m^{8} \text {, } \\
& a_{14,0}=\frac{13}{729} a_{18,0}{ }^{3}, \\
& a_{14,2}=\frac{34}{9} m^{2} a_{18,0}{ }^{2}, \\
& a_{14,4}=\frac{460}{9} m^{4} a_{18,0} \text {, } \\
& a_{14,6}=120 m^{6} \text {, } \\
& a_{16,0}=\frac{41}{180} a_{18,0}{ }^{2}, \\
& a_{16,2}=\frac{101}{9} m^{2} a_{18,0}, \\
& a_{16,4}=45 m^{4} \text {, } \\
& a_{18,2}=10 \mathrm{~m}^{2} \text {, } \\
& b_{0,0}=\frac{37349}{344373768000} b_{0,12} a_{18,0}{ }^{6} \text {, } \\
& b_{0,2}=\frac{93301}{3188646000} b_{0,12} a_{18,0}{ }^{5} \text {, } \\
& b_{0,4}=-\frac{6097}{23619600} b_{0,12} a_{18,0}{ }^{4}, \\
& b_{0,6}=\frac{23}{21870} b_{0,12} a_{18,0}{ }^{3},
\end{aligned}
$$




$$
\begin{aligned}
& b_{0,8}=\frac{5}{972} b_{0,12} a_{18,0}{ }^{2}, \\
& b_{0,10}=\frac{1}{3} b_{0,12} a_{18,0}, \\
& b_{2,0}=-\frac{1183}{70858800} b_{0,12} a_{18,0}{ }^{5} \mathrm{~m}^{2} \text {, } \\
& b_{2,2}=\frac{5243}{11809800} b_{0,12} a_{18,0}{ }^{4} \mathrm{~m}^{2} \text {, } \\
& b_{2,4}=-\frac{131}{21870} b_{0,12} a_{18,0}{ }^{3} m^{2}, \\
& b_{2,6}=-\frac{23}{243} b_{0,12} a_{18,0}{ }^{2} \mathrm{~m}^{2}, \\
& b_{2,8}=-m^{2} b_{0,12} a_{18,0} \text {, } \\
& b_{2,10}=-2 m^{2} b_{0,12} \text {, } \\
& b_{4,0}=-\frac{7033}{23619600} m^{4} b_{0,12} a_{18,0}{ }^{4} \text {, } \\
& b_{4,2}=\frac{209}{21870} m^{4} b_{0,12} a_{18,0}{ }^{3} \text {, } \\
& b_{4,4}=-\frac{113}{486} m^{4} b_{0,12} a_{18,0}{ }^{2} \text {, } \\
& b_{4,6}=-\frac{146}{45} b_{0,12} m^{4} a_{18,0}, \\
& b_{4,8}=-9 m^{4} b_{0,12} \text {, } \\
& b_{6,0}=-\frac{19}{153090} m^{6} b_{0,12} a_{18,0}{ }^{3} \text {, } \\
& b_{6,2}=\frac{1307}{8505} m^{6} b_{0,12} a_{18,0}{ }^{2} \text {, } \\
& b_{6,4}=\frac{26}{63} m^{6} b_{0,12} a_{18,0}, \\
& b_{6,6}=-\frac{36}{7} m^{6} b_{0,12} \text {, } \\
& b_{8,0}=\frac{1}{2268} m^{8} b_{0,12} a_{18,0}{ }^{2}, \\
& b_{8,2}=\frac{131}{189} b_{0,12} a_{18,0} m^{8}, \\
& b_{8,4}=\frac{25}{7} m^{8} b_{0,12} \text {, } \\
& b_{10,0}=-\frac{1}{189} m^{10} b_{0,12} a_{18,0} . \\
& b_{10,2}=\frac{18}{7} m^{10} b_{0,12} \text {, } \\
& b_{12,0}=-\frac{1}{7} m^{12} b_{0,12} \text {, } \\
& c_{0,0}=-\frac{199927}{1721868840000} c_{12,0} a_{18,0}{ }^{6}, \\
& c_{0,2}=-\frac{878003}{3188646000} c_{12,0} a_{18,0}{ }^{5} \mathrm{~m}^{2}, \\
& c_{0,4}=-\frac{84329}{23619600} m^{4} a_{18,0}{ }^{4} c_{12,0},
\end{aligned}
$$




$$
\begin{aligned}
& c_{0,6}=-\frac{77}{2430} m^{6} a_{18,0}{ }^{3} c_{12,0} \text {, } \\
& c_{0,8}=-\frac{203}{972} m^{8} a_{18,0}{ }^{2} c_{12,0}, \\
& c_{0,10}=-\frac{49}{45} c_{12,0} a_{18,0} m^{10}, \\
& c_{0,12}=-7 m^{12} c_{12,0} \text {, } \\
& c_{2,0}=\frac{57967}{1062882000} a_{18,0}{ }^{5} c_{12,0} \text {, } \\
& c_{2,2}=\frac{7511}{2361960} c_{12,0} a_{18,0}{ }^{4} \mathrm{~m}^{2}, \\
& c_{2,4}=\frac{161}{2430} m^{4} a_{18,0}{ }^{3} c_{12,0} \text {, } \\
& c_{2,6}=\frac{497}{243} m^{6} a_{18,0}{ }^{2} c_{12,0}, \\
& c_{2,8}=7 c_{12,0} a_{18,0} m^{8}, \\
& c_{2,10}=14 m^{10} c_{12,0} \text {, } \\
& c_{4,0}=-\frac{1981}{4723920} c_{12,0} a_{18,0}{ }^{4}, \\
& c_{4,2}=-\frac{371}{21870} c_{12,0} a_{18,0}{ }^{3} \mathrm{~m}^{2}, \\
& c_{4,4}=-\frac{49}{486} m^{4} c_{12,0} a_{18,0}{ }^{2}, \\
& c_{4,6}=\frac{182}{9} c_{12,0} a_{18,0} m^{6}, \\
& c_{4,8}=63 m^{8} c_{12,0} \text {, } \\
& c_{6,0}=\frac{47}{21870} c_{12,0} a_{18,0}{ }^{3}, \\
& c_{6,2}=-\frac{443}{1215} a_{18,0}{ }^{2} c_{12,0} m^{2}, \\
& c_{6,4}=-\frac{58}{9} c_{12,0} m^{4} a_{18,0}, \\
& c_{6,6}=36 m^{6} c_{12,0} \text {, } \\
& c_{8,0}=-\frac{11}{972} a_{18,0}{ }^{2} c_{12,0}, \\
& c_{8,2}=-\frac{187}{27} m^{2} a_{18,0} c_{12,0}, \\
& c_{8,4}=-25 m^{4} c_{12,0}, \\
& c_{10,0}=\frac{37}{135} c_{12,0} a_{18,0} \text {, } \\
& c_{10,2}=-18 m^{2} c_{12,0} \text {, } \\
& b=-\frac{1}{270} m^{2} a_{18,0} \text {. }
\end{aligned}
$$

This set leads to a 8-lump solution to (1)

$$
u(x, y, t)=\frac{6 b}{a}(\ln f)_{X X},
$$

where $X=x+m t$, the coefficients of $f$ are determined by the above constraining equations, and $m, a, b, \alpha$, and $\beta$ are arbitrary real constants. When setting $m=2, a=-2$, 


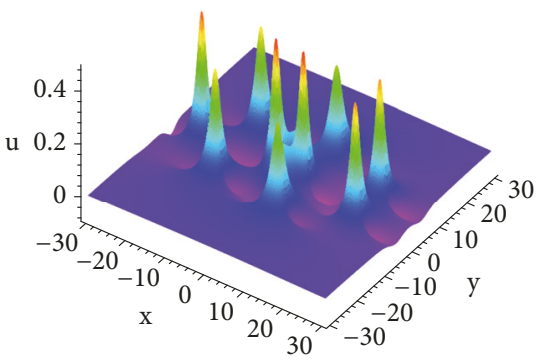

(a)

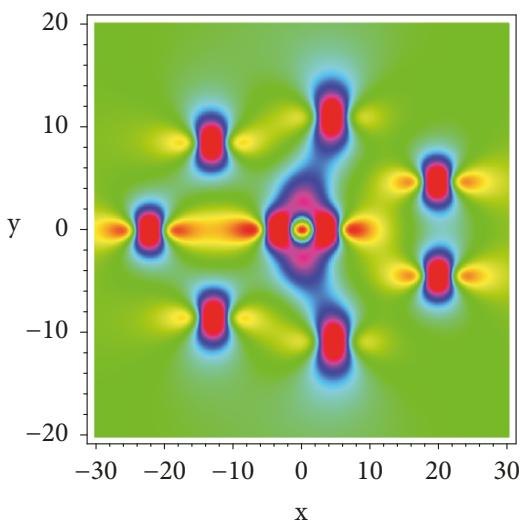

(d)

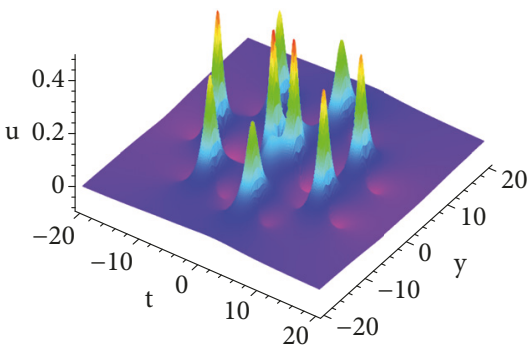

(b)

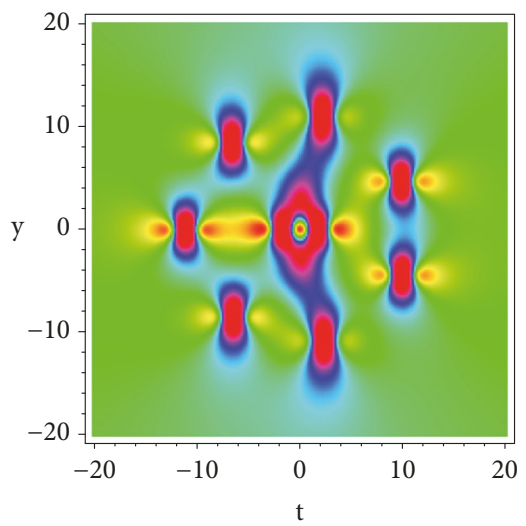

(e)

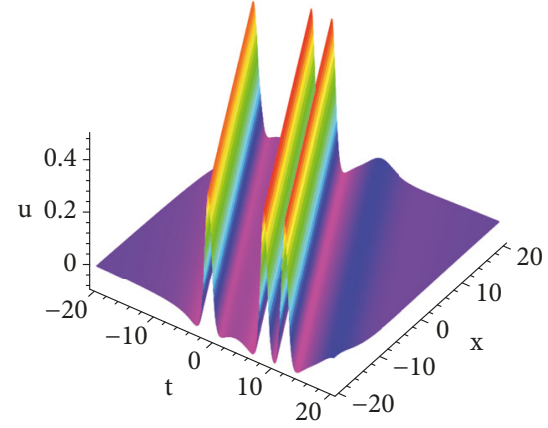

(c)

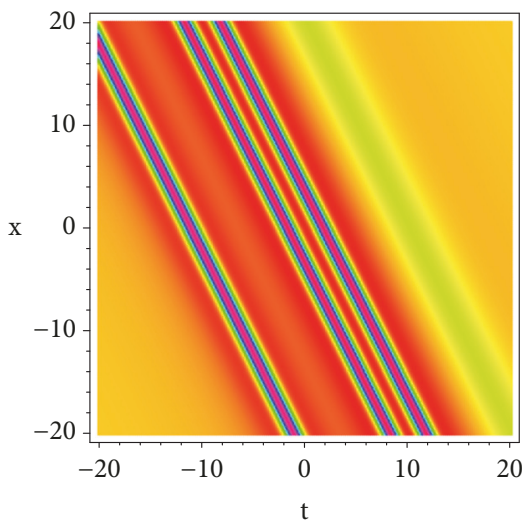

(f)

Figure 8: Plots (colored online) of 8-lump wave $u$ (21) with $m=2, a=-2, \alpha=3 \times 10^{9}, \beta=4 \times 10^{9}, b_{0,12}=c_{12,0}=1, a_{18,0}=600$. (a) Perspective view of the wave $u(x, y, 0)$, (b) perspective view of the wave $u(0, y, t)$, (c) perspective view of the wave $u(x, 0, t)$, (d) overhead view of the wave (a), (e) overhead view of the wave (b), and (f) overhead view of the wave (c).

$\alpha=3 \times 10^{9}, \beta=4 \times 10^{9}, b_{0,12}=c_{12,0}=1, a_{18,0}=600$, we find that solution (21) (Figures 8(a), 8(b), 8(d), and 8(e)) possesses eight lump waves, namely, seven single lump waves and one second order rogue wave $u_{2}$ (see Figures $2(\mathrm{a})$ and 2(b)). The seven single lump waves array a heptagon around the second order rogue wave. If we set $y=0$, the 8-lump solution develops into four-soliton solution, which is plotted in Figures 8(c) and 8(f). Figures 9(a) and 9(e) show that the 8 -lump wave develops into fourth order rogue wave $u_{4}$ of Section 2 when $\alpha^{2}+\beta^{2}$ declines to 0 . From Figures 9(b)-9(d) and $9(f)-9(h)$, we can observe that the fouth order rogue wave splits into a set of seven first order rogue waves and one second order rogue as the center with parameters $\alpha$ and $\beta$ gradually become large enough.

\section{Conclusions}

In this paper, we focus on investigating the rogue wave solutions and the rogue type multiple lump solutions of the $(2+1)$-dimensional Benjamin-Ono equation by means of the bilinear method. Based on the reduced bilinear equations, four kinds of rogue wave solutions are obtained. The number of the higher peaks of the multiple rogue wave solutions is equivalent to the subscript of $u_{n}$. The maxima of the solutions $u_{n}$ all lie on the line $y=0$. The multiple rogue waves develop into the multiple stripe solitons in the plane $(t, x)$. A theorem for constructing multiple lump solutions has been presented by introducing three polynomial functions $F_{n}, P_{n}$, and $Q_{n}(10)$ of degree $n(n+1)$. The multiple lump waves tend to the low order rogue waves as the parameter $\alpha^{2}+\beta^{2}$ is near 0 . Numerical experiments show that the multiple lump solutions will produce new peaks with the increase of parameters. The number of the lumps will be fixed and all peaks will tend to the same height when $\alpha^{2}+$ $\beta^{2}$ is sufficiently large. The method presented in this paper shows that Hirota's bilinear method is a powerful approch to construct the high order rational solutions to nonlinear integrable equations. Based on the bilinear forms, one can construct different types of rational solutions by choosing different polynomial functions. The results might be helpful in explaining nonlinear wave phenomena in fluid mechanics.

\section{Data Availability}

The data used to support the findings of this study are included within the article.

\section{Conflicts of Interest}

The authors declare that they have no conflicts of interest. 


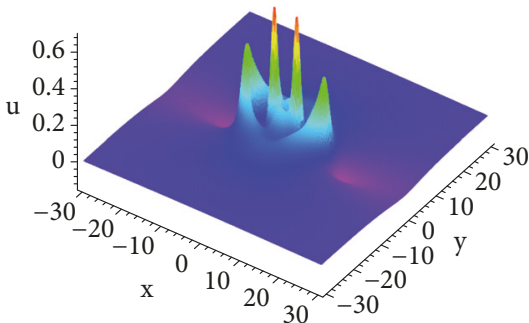

(a)

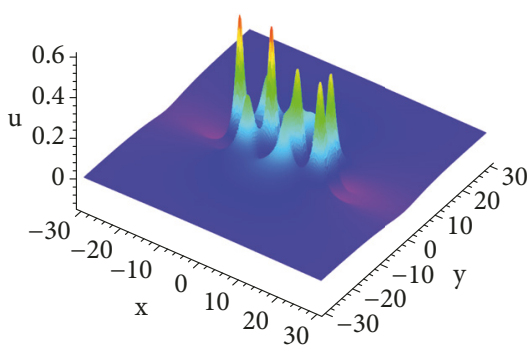

(c)

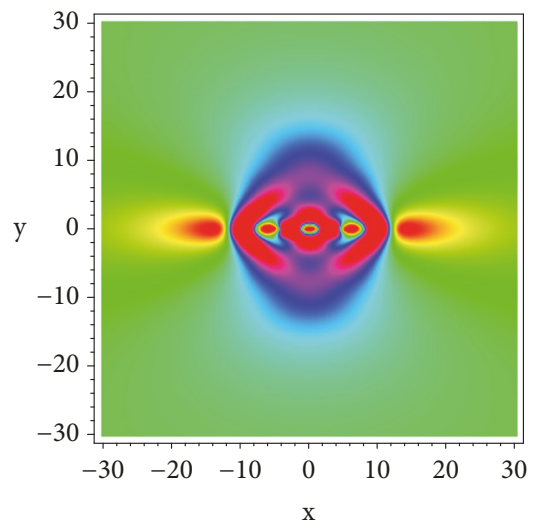

(e)

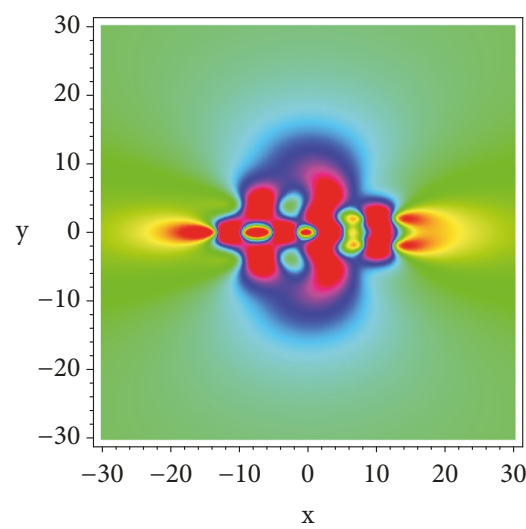

(g)

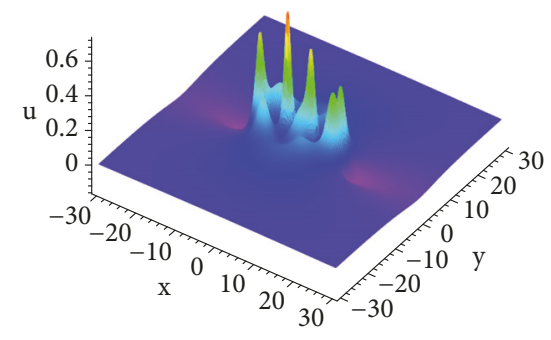

(b)

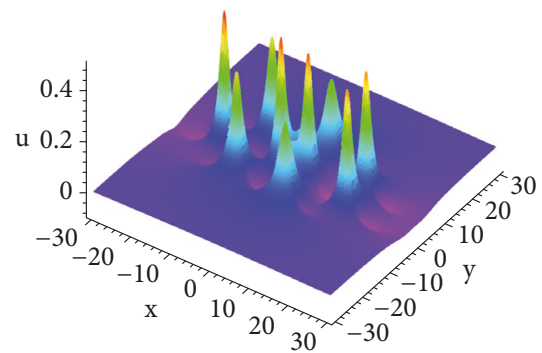

(d)

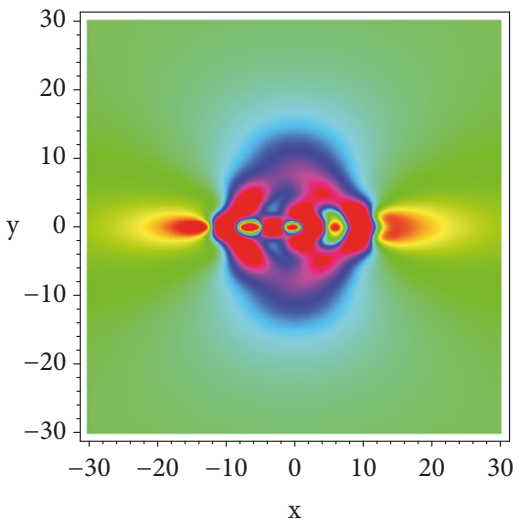

(f)

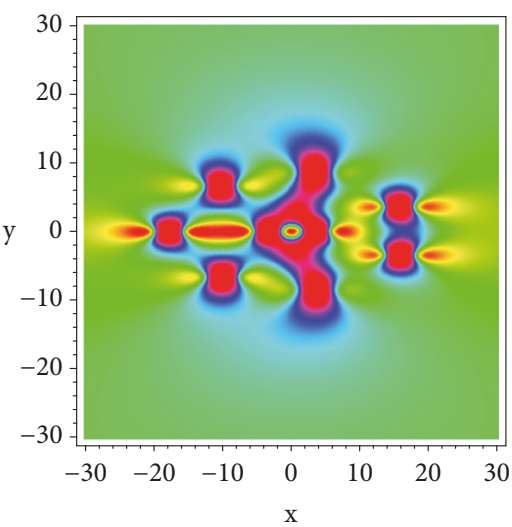

(h)

FIGURE 9: Plots (colored online) of 8-lump wave $u$ (21) with $m=2, a=-2, b_{0,12}=c_{12,0}=1, a_{18,0}=600$. (a) Perspective view of the wave $u(x, y, 0)$ with $\alpha=30, \beta=40$, (b) perspective view of the wave $u(x, y, 0)$ with $\alpha=1 \times 10^{7}, \beta=2 \times 10^{7}$, (c) perspective view of the wave $u(x, y, 0)$ with $\alpha=5 \times 10^{7}, \beta=7 \times 10^{7}$, (d) perspective view of the wave $u(x, y, 0)$ with $\alpha=8 \times 10^{8}, \beta=1 \times 10^{9}$, (e) overhead view of the wave (a), (f) overhead view of the wave (b), (g) overhead view of the wave (c), and (h) overhead view of the wave (d). 


\section{Acknowledgments}

This research is supported by Scientific and Technologial Innovation Programs of Higher Education Institutions in Shanxi, Initial Scientific Research Fund of the High-level Talents in 2019 in North University of China (No. 11012411), and the Fund for Shanxi "1331KIRT".

\section{References}

[1] M. Rudman and P. W. Cleary, "The influence of mooring system in rogue wave impact on an offshore platform," Ocean Engineering, vol. 115, no. 15, pp. 168-181, 2016.

[2] F. Fedele, "Rogue waves in oceanic turbulence," Physica D: Nonlinear Phenomena, vol. 237, no. 14-17, pp. 2127-2131, 2008.

[3] R. Grimshaw, E. Pelinovsky, T. Taipova, and A. Sergeeva, "Rogue internal waves in the ocean: Long wave model," The European Physical Journal Special Topics, vol. 185, no. 1, pp. 195-208, 2010.

[4] C. Bayındır and F. Ozaydin, "Freezing optical rogue waves by Zeno dynamics," Optics Communications, vol. 413, no. 15, pp. 141-146, 2018.

[5] J. S. He, E. G. Charalampidis, P. G. Kevrekidis, and D. J. Frantzeskakis, "Rogue waves in nonlinear Schrödinger models with variable coefficients: application to bose-einstein condensates," Physics Letters A, vol. 378, no. 5-6, pp. 577-583, 2014.

[6] L. C. Zhao, "Dynamics of nonautonomous rogue waves in Bose-Einstein condensate," Annals of Physics, vol. 329, pp. 7379, 2013.

[7] Z. Y. Yan, "Financial rogue waves," Communications in Theoretical Physics, vol. 54, no. 5, pp. 947-949, 2010.

[8] Z. Y. Yan, "Vector financial rogue waves," Physics Letters A, vol. 375, no. 48, pp. 4274-4279, 2011.

[9] B. L. Guo, L. M. Ling, and Q. P. Liu, "Nonlinear schrödinger equation: generalized darboux transformation and rogue wave solutions," Physical Review E: Statistical, Nonlinear, and Soft Matter Physics, vol. 85, no. 2, Article ID 026607, 2012.

[10] J. S. He, L. H. Wang, L. J. Li, K. Porsezian, and R. Erdélyi, "Fewcycle optical rogue waves: Complex modified Korteweg-de Vries equation," Physical Review E: Statistical, Nonlinear, and Soft Matter Physics, vol. 89, no. 6, Article ID 062917, 2014.

[11] W. X. Ma, Y. Zhou, and R. Dougherty, "Lump-type solutions to nonlinear differential equations derived from generalized bilinear equations," International Journal of Modern Physics B, vol. 30, no. 28-29, Article ID 1640018, 2016.

[12] W. X. Ma and Y. Zhou, "Lump solutions to nonlinear partial differential equations via Hirota bilinear forms," Journal of Differential Equations, vol. 264, no. 4, pp. 2633-2659, 2018.

[13] W. X. Ma, "Lump solutions to the Kadomtsev-Petviashvili equation," Physics Letters A, vol. 379, no. 36, pp. 1975-1978, 2015.

[14] H. Q. Zhang and W. X. Ma, "Lump solutions to the $(2+1)-$ dimensional Sawada-Kotera equation," Nonlinear Dynamics, vol. 87, no. 4, pp. 2305-2310, 2017.

[15] Z. L. Zhao, Y. Chen, and B. Han, "Lump soliton, mixed lump stripe and periodic lump solutions of a $(2+1)$-dimensional asymmetrical Nizhnik-Novikov-Veselov equation," Modern Physics Letters B, vol. 31, no. 14, Article ID 1750157, 2017.

[16] Z. L. Zhao and B. Han, "Lump solutions of a (3+1)-dimensional B-type KP equation and its dimensionally reduced equations," Analysis and Mathematical Physics, vol. 9, no. 1, pp. 119-130, 2019.
[17] S. T. Chen and W. X. Ma, "Lump solutions of a generalized Calogero-Bogoyavlenskii-Schiff equation," Computers \& Mathematics with Applications, vol. 76, no. 7, pp. 1680-1685, 2018.

[18] X. Lü, S. T. Chen, and W. X. Ma, "Constructing lump solutions to a generalized Kadomtsev-Petviashvili-Boussinesq equation," Nonlinear Dynamics, vol. 86, no. 1, pp. 523-534, 2016.

[19] X. M. Zhu, D. J. Zhang, and D. Y. Chen, "Lump solutions of kadomtsev-petviashvili I equation in non-uniform media," Communications in Theoretical Physics, vol. 55, no. 1, pp. 13-19, 2011.

[20] B. Ren, W. X. Ma, and J. Yu, "Rational solutions and their interaction solutions of the (2+1)-dimensional modified dispersive water wave equation," Computers \& Mathematics with Applications, vol. 77, no. 8, pp. 2086-2095, 2019.

[21] Z. L. Zhao and B. Han, "Lie symmetry analysis, Bäcklund transformations, and exact solutions of a $(2+1)$-dimensional BoitiLeon-Pempinelli system," Journal of Mathematical Physics, vol. 58, no. 10, Article ID 101514, 2017.

[22] Z. L. Zhao and B. Han, "Residual symmetry, Bäcklund transformation and CRE solvability of a (2+1)-dimensional nonlinear system," Nonlinear Dynamics, vol. 94, no. 1, pp. 461-474, 2018.

[23] Z. L. Zhao, "Bäcklund transformations, rational solutions and soliton-cnoidal wave solutions of the modified Kadomtsev-Petviashvili equation," Applied Mathematics Letters, vol. 89, pp. 103-110, 2019.

[24] Z. L. Zhao and B. Han, "Nonlocal symmetry and explicit solutions from the CRE method of the Boussinesq equation," The European Physical Journal Plus, vol. 133, no. 4, Article ID $144,2018$.

[25] Z. L. Zhao and B. Han, "Lie symmetry analysis of the Heisenberg equation," Communications in Nonlinear Science and Numerical Simulation, vol. 45, pp. 220-234, 2017.

[26] Z. L. Zhao and B. Han, "On symmetry analysis and conservation laws of the AKNS system," Zeitschrift für Naturforschung A: A Journal of Physical Sciences, vol. 71, pp. 741-750, 2016.

[27] Z. L. Zhao and B. Han, "On optimal system, exact solutions and conservation laws of the Broer-Kaup system," The European Physical Journal Plus, vol. 130, no. 11, Article ID 223, 2015.

[28] L. J. Guo, L. H. Wang, Y. Cheng, and J. S. He, "Highorder rogue wave solutions of the classical massive Thirring model equations," Communications in Nonlinear Science and Numerical Simulation, vol. 52, pp. 11-23, 2017.

[29] P. G. Estévez and J. Prada, "Algorithmic construction of lumps," Theoretical and Mathematical Physics, vol. 151, no. 3, pp. 744-751, 2007.

[30] A. S. Fokas, D. E. Pelinovsky, and C. Sulem, "Interaction of lumps with a line soliton for the DSII equation," Physica D: Nonlinear Phenomena, vol. 152-153, pp. 189-198, 2001.

[31] Y. N. Tang, S. Q. Tao, and Q. Guan, "Lump solitons and the interaction phenomena of them for two classes of nonlinear evolution equations," Computers \& Mathematics with Applications, vol. 72, no. 9, pp. 2334-2342, 2016.

[32] Y. Zhou, S. Manukure, and W. X. Ma, "Lump and lump-soliton solutions to the Hirota-Satsuma-Ito equation," Communications in Nonlinear Science and Numerical Simulation, vol. 68, pp. 5662, 2019.

[33] J. G. Rao, D. Mihalache, Y. Cheng, and J. S. He, "Lump-soliton solutions to the Fokas system," Physics Letters A, vol. 383, no. 11, pp. 1138-1142, 2019.

[34] J. Y. Yang, W. X. Ma, and Z. Y. Qin, "Lump and lump-soliton solutions to the (2+1)-dimensional Ito equation," Analysis and Mathematical Physics, vol. 8, no. 3, pp. 427-436, 2018. 
[35] C. H. He, Y. N. Tang, and J. L. Ma, "New interaction solutions for the (3+1)-dimensional Jimbo-Miwa equation," Computers \& Mathematics with Applications, vol. 76, no. 9, pp. 2141-2147, 2018.

[36] Y. Zhang, Y. P. Liu, and X. Y. Tang, "M-lump solutions to a (3+1)-dimensional nonlinear evolution equation," Computers \& Mathematics with Applications, vol. 76, no. 3, pp. 592-601, 2018.

[37] W. C. Hu, W. H. Huang, Z. M. Lu, and Y. Stepanyants, "Interaction of multi-lumps within the Kadomtsev-Petviashvili equation," Wave Motion, vol. 77, pp. 243-256, 2018.

[38] B. Q. Li and Y. L. Ma, "Multiple-lump waves for a $(3+1)$ dimensional Boiti-Leon-Manna-Pempinelli equation arising from incompressible fluid," Computers \& Mathematics with Applications, vol. 76, no. 1, pp. 204-214, 2018.

[39] B. N. Sun and A. M. Wazwaz, "General high-order breathers and rogue waves in the $(3+1)$-dimensional KP-Boussinesq equation," Communications in Nonlinear Science and Numerical Simulation, vol. 64, pp. 1-13, 2018.

[40] Zhaqilao, "A symbolic computation approach to constructing rogue waves with a controllable center in the nonlinear systems," Computers \& Mathematics with Applications, vol. 75, no. 9, pp. 3331-3342, 2018.

[41] W. Y. Cui and Zhaqilao, "Multiple rogue wave and breather solutions for the (3+1)-dimensional KPI equation," Computers \& Mathematics with Applications, vol. 76, no. 5, pp. 1099-1107, 2018.

[42] Z. L. Zhao and L. C. He, "Multiple lump solutions of the (3+1)-dimensional potential Yu-Toda-Sasa-Fukuyama equation," Applied Mathematics Letters, vol. 95, pp. 114-121, 2019.

[43] Y. K. Liu, B. Li, and H. L. An, "General high-order breathers, lumps in the (2+1)-dimensional Boussinesq equation," Nonlinear Dynamics, vol. 92, no. 4, pp. 2061-2076, 2018.

[44] E. G. Fan, Integrable System and Computer Algebra, Science Press, Beijing, China, 2004.

[45] A. Esfahani, "Remarks on solitary waves of the generalized two dimensional Benjamin-Ono equation," Applied Mathematics and Computation, vol. 218, no. 2, pp. 308-323, 2011. 


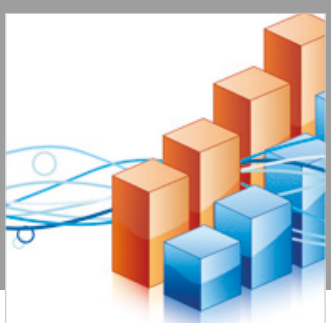

Advances in

Operations Research

\section{-n-m}
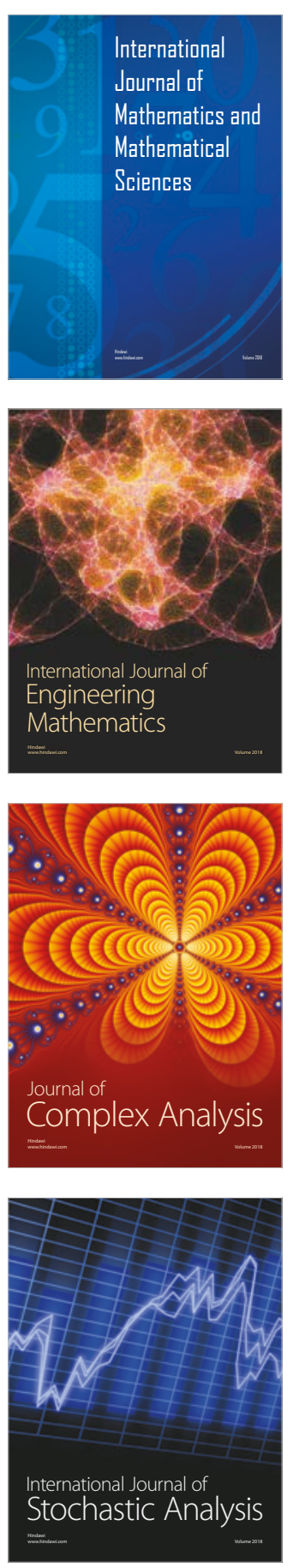
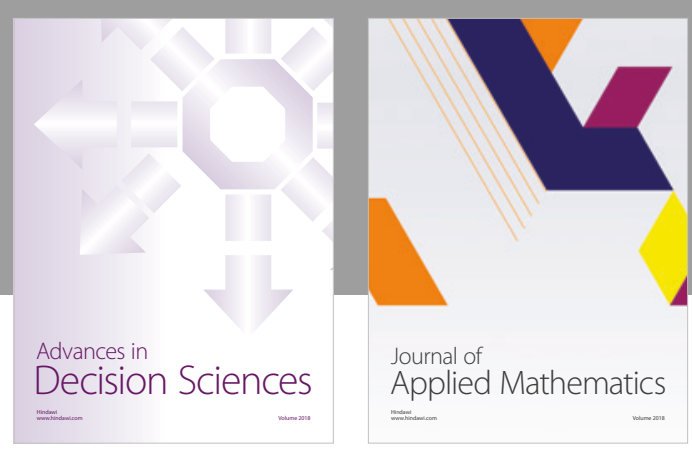

Journal of

Applied Mathematics
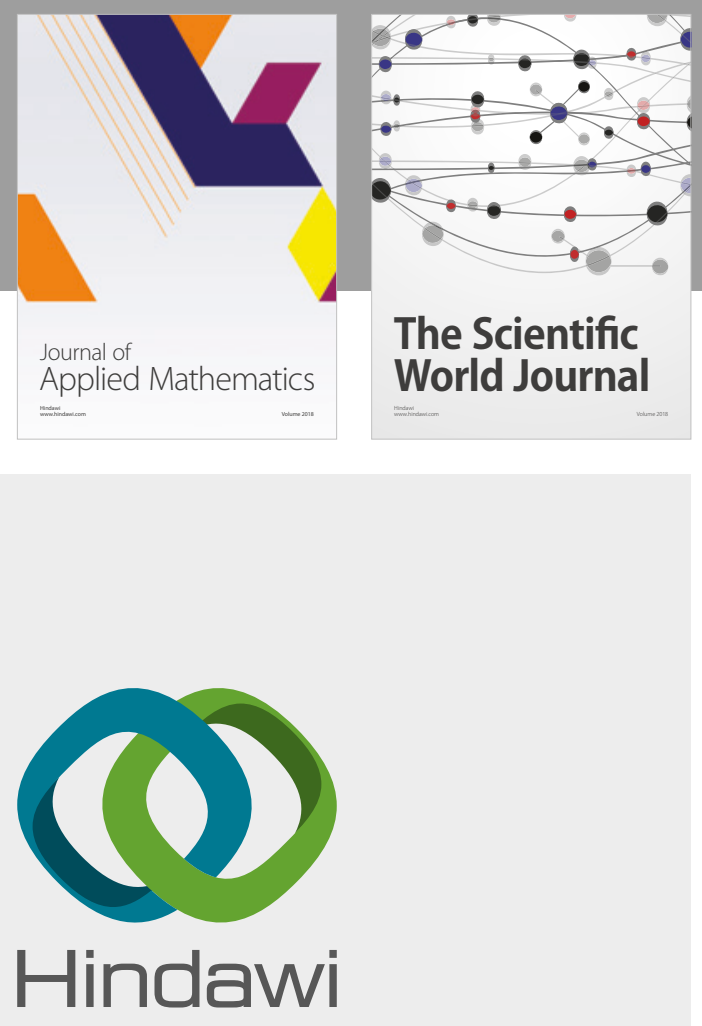

Submit your manuscripts at

www.hindawi.com

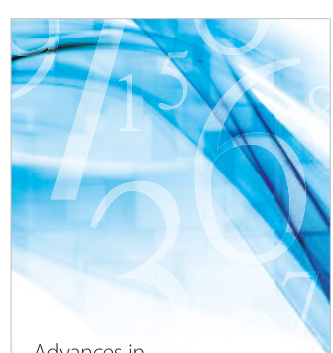

Advances in
Numerical Analysis
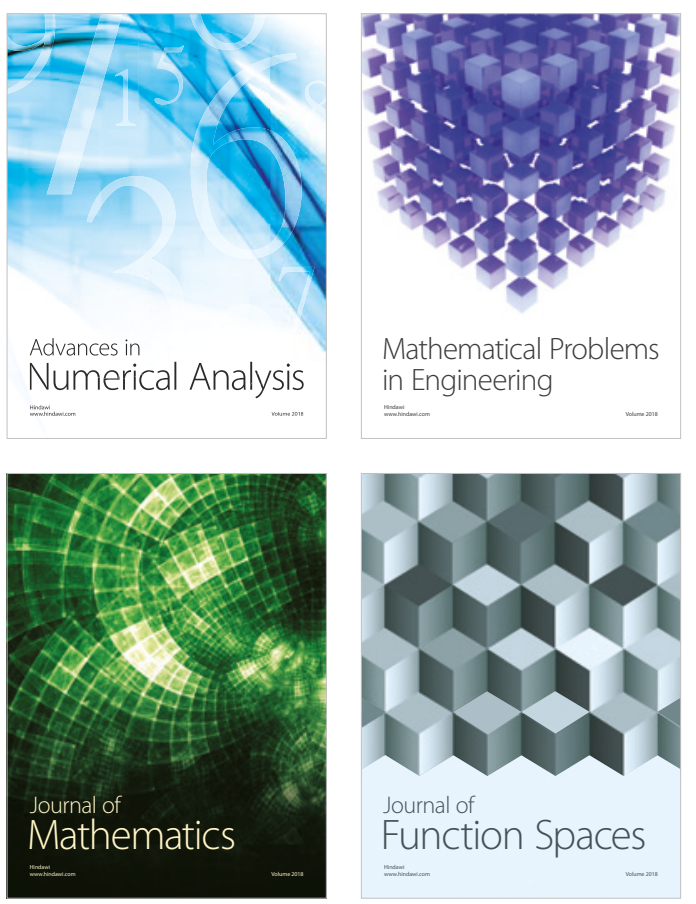

Mathematical Problems in Engineering

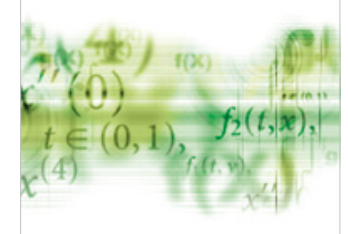

International Journal of

Differential Equations

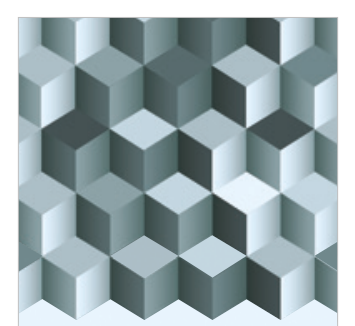

Journal of

Function Spaces
The Scientific

World Journal

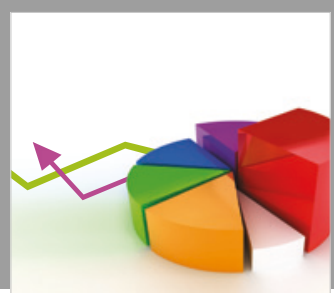

Journal of

Probability and Statistics
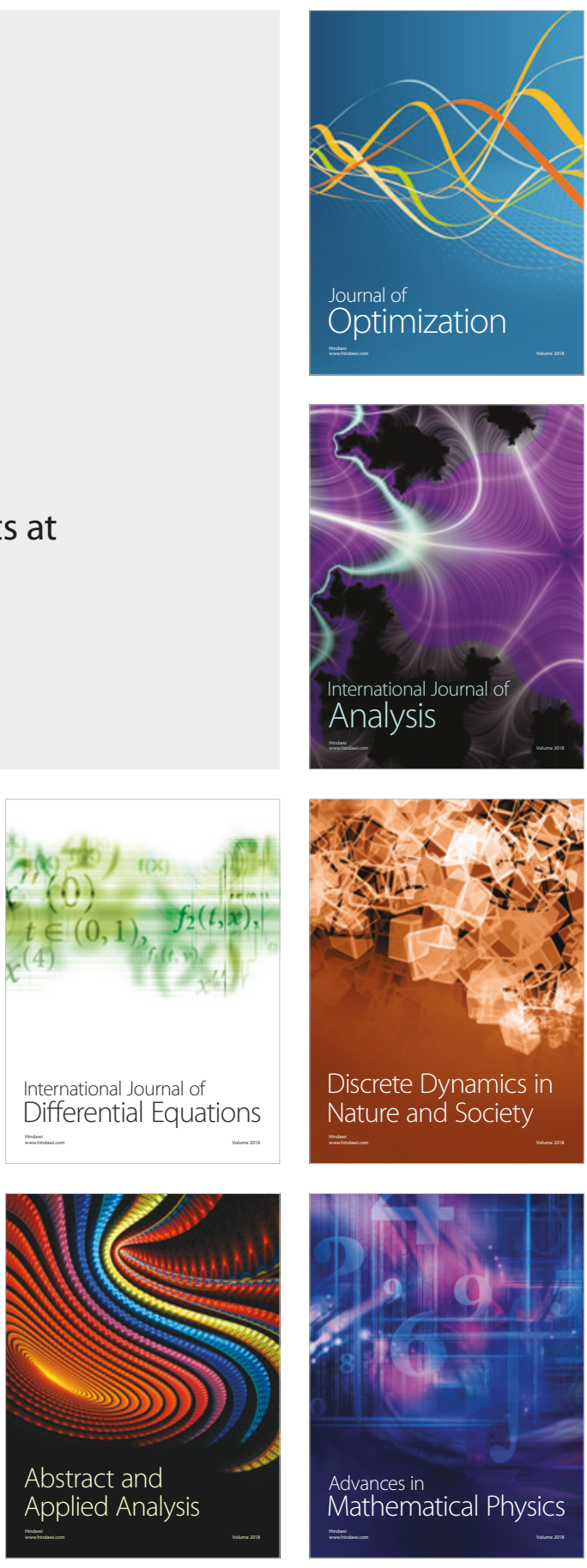